\title{
STRUCTURE OF THE ACCRETION FLOW IN BROAD-LINE RADIO GALAXIES: THE CASE OF 3C 390.3
}

\author{
R. M. Sambruna ${ }^{1}$, J. N. Reeves ${ }^{2,3}$, V. Braito ${ }^{4,5}$, K. T. Lewis $^{2,6}$, M. Eracleous ${ }^{7,8}$, M. Gliozzi ${ }^{9}$, F. Tavecchio ${ }^{10}$, \\ D. R. Ballantyne ${ }^{11}$, P. M. Ogle ${ }^{12}$, A. J. Barth ${ }^{13}$, AND J. Tueller ${ }^{1}$ \\ ${ }^{1}$ NASA/GSFC, Code 661, Greenbelt, MD 20771, USA \\ 2 NASA/GSFC, Code 662, Greenbelt, MD 20771, USA \\ ${ }^{3}$ Astrophysics Group, School of Physical \& Geographical Sciences, Keele University, Keele, Staffordshire ST5 5BG, UK \\ ${ }^{4}$ Department of Physics and Astronomy, Johns Hopkins University, Baltimore, MD 21218, USA \\ ${ }^{5}$ University of Leicester, Department of Physics \& Astronomy, University Road, Leicester LE1 7RH, UK \\ ${ }^{6}$ Dickinson College, Department of Physics and Astronomy, Carlisle, PA 17013, USA \\ ${ }^{7}$ Department of Astronomy \& Astrophysics, The Pennsylvania State University, 525 Davey Lab, University Park, PA 16802, USA \\ ${ }^{8}$ Center for Gravitational Wave Physics, The Pennsylvania State University, University Park, PA 16802, USA \\ ${ }^{9}$ George Mason University, Department of Physics and Astronomy, 4400 University Dr., Fairfax, VA 22030, USA \\ ${ }^{10}$ INAF, Osserv. Astron. di Brera, via Bianchi 46, I-23807 Merate, Italy \\ ${ }^{11}$ Center for Relativistic Astrophysics, School of Physics, Georgia Institute of Technology, 837 State Street, Atlanta, GA 30332, USA \\ 12 Spitzer Science Center, California Institute of Technology, Mail Code 220-6, Pasadena, CA 91125, USA \\ 13 Department of Physics and Astronomy, 4129 Frederick Reines Hall, University of California, Irvine, CA 92697-4575, USA \\ Received 2009 January 2; accepted 2009 June 2; published 2009 July 14
}

\begin{abstract}
We present XMM-Newton and Suzaku observations of the broad-line radio galaxy 3C 390.3 acquired in 2004 October and 2006 December, respectively. An archival Swift BAT spectrum from the 9 month survey is also analyzed, as well as an optical spectrum simultaneous to XMM-Newton. At soft X-rays, no absorption features are detected in the Reflection Grating Spectrometer spectrum of 3C 390.3; a narrow emission line is found at $0.564 \mathrm{keV}$, most likely originating in the narrow-line region. Both the EPIC and XIS data sets confirm the presence of an Fe K $\alpha$ emission line at $6.4 \mathrm{keV}$ with equivalent width $(\mathrm{EW})=40 \mathrm{eV}$. The $\mathrm{Fe} \mathrm{K} \alpha$ line has a width FWHM $\sim 8800 \mathrm{~km} \mathrm{~s}^{-1}$, consistent within a factor of 2 with the width of the double-peaked $\mathrm{H} \alpha$ line, suggesting an origin from the broad-line region. The data show for the first time a weak, broad bump extending from 5 to $7 \mathrm{keV}$. When fitted with a Gaussian, its centroid energy is $6.6 \mathrm{keV}$ in the source's rest frame with FWHM of $43,000 \mathrm{~km} \mathrm{~s}^{-1}$ and EW of $50 \mathrm{eV}$; its most likely interpretation is emission from He-like Fe (Fe XXV), suggesting the presence of an ionized medium in the inner regions of 3C 390.3. The broadband $0.5-100 \mathrm{keV}$ continuum is well described by a single power law with photon index $\Gamma=1.6$ and cutoff energy $157 \mathrm{keV}$, plus cold reflection with strength $R=0.5$. In addition, ionized reflection is required to account for the $6.6 \mathrm{keV}$ bump in the broadband continuum, yielding an ionization parameter $\xi \sim 2700 \mathrm{erg} \mathrm{cm} \mathrm{s}^{-1}$; the inner radius of the ionized reflector is constrained to be larger than $20 r_{G}$, although this result depends on the assumed emissivity profile of the disk. If true, we argue that the lack of broad $\mathrm{Fe} \mathrm{K}$ emission from within $20 r_{G}$ indicates that the innermost regions of the disk in 3C 390.3 are obscured and/or poorly illuminated. While the spectral energy distribution (SED) of 3C 390.3 is generally dominated by accretionrelated continuum, during accretion low states the jet can significantly contribute in the optical to X-ray bands via synchrotron self-Compton emission. The Compton component is expected to extend to and peak at GeV gamma rays where it will be detected with the Fermi Gamma-Ray Space Telescope during its first few years of operation.
\end{abstract}

Key words: galaxies: active - galaxies: individual (3C390.3) - galaxies: jets - galaxies: nuclei - galaxies: Seyfert

Online-only material: color figures

\section{INTRODUCTION}

According to a widely accepted view active galactic nuclei (AGNs), including radio-loud sources, are ultimately powered by accretion onto a central supermassive black hole (e.g., Blandford 1985). A relativistic jet originates in the innermost regions and transports energy and momentum to the more distant lobes of the radio galaxy. The jet angle-defined as the angle between the jet axis and the line of sight-increases from blazars to broad-line radio galaxy (BLRG) and narrow-line radio galaxy, in which the direct view to the center is obscured by thick matter surrounding the nucleus. As the jet angle increases, the importance of its emission decreases, due to beaming effects.

Previous X-ray observations of BLRGs established that these sources exhibit Seyfert-like spectra with subtle but significant differences. Specifically, the X-ray continua of BLRGs are flatter $(\langle\Gamma\rangle \approx 1.7$; Zdziarski \& Grandi 2001), and their reflection features weaker than their radio-quiet cousins (e.g., Sambruna et al. 2002; Ballantyne 2007, and references therein). To account for these properties, it was suggested that the reflection features from a standard, cold disk similar to Seyferts are weak in BLRGs because they are diluted by the nonthermal beamed jet emission (Grandi et al. 2002). Another possibility is that the reprocessing medium in radio-loud objects subtends a smaller solid angle to the central X-ray source, as in ion torus/advectiondominated accretion-flow (ADAF) models (Rees et al. 1982), as advocated by Eracleous et al. (2000). Alternatively, BLRGs could have more highly ionized accretion disks than Seyfert 1s, as a result of higher accretion rates (Ballantyne et al. 2002). This model successfully fitted the XMM-Newton spectrum of 3C 120 (Ballantyne et al. 2004). However, fitting the same data set Ogle et al. (2000) found no evidence for broad Fe K emission line, and concluded based on the line width and equivalent width (EW) that the $\mathrm{Fe} \mathrm{K} \alpha$ line originates from the optical broad-line region (BLR). The various scenarios discussed above are not mutually exclusive, however, and it is possible that jet dilution could 
affect the strength of the spectroscopic signature of an ionized disk, for example. However, from the analysis of BeppoSAX observations of BLRGs, Grandi \& Palumbo (2007) concluded that if present, the jet contributes minimally to the $2-10 \mathrm{keV}$ emission but should dominate at $\mathrm{GeV}$ gamma rays.

The key observational features required to disentangle the various possibilities are broadband coverage and sensitivity at medium-hard X-rays, where the reflection features and the jet nonthermal emission mix. Previous attempts at acquiring broadband coverage at these energies by coordinating $X M M-N e w t o n$ and RXTE had modest success due to the different scheduling constraints of the two missions, while BeppoSAX had limited sensitivity in the $\mathrm{Fe} \mathrm{K}$ line region. It was not until the advent of Suzaku that the basic requisite of simultaneous broadband coverage and larger collecting area in 6-7 keV was achieved. Thus, we began a program of observations of a number of "classical" (i.e., bright) BLRGs with Suzaku (3C 390.3, 3C 382, 3C 445, 3C 111); two additional sources, 3C 120 and $4 \mathrm{C}+74.26$, are already in the Suzaku archive (Kataoka et al. 2007; Larsson et al. 2008).

Our program consists of two complementary goals: (1) determine if jet dilution is responsible for the weakness of the reflection features in BLRGs and (2) study the structure of the accretion flow in radio-loud AGN, to understand which conditions favor the formation of powerful, relativistic jets. The source whose Suzaku observations are presented here, 3C 390.3, is an optimal candidate for goal 2. As described in Section 2 and shown in Section 7.3, the jet does not make a significant contribution to the nuclear X-ray emission of 3C 390.3, and as such this BLRG qualifies as an optimal laboratory for investigating the accretion-flow structure in radio-loud AGN.

In this paper, we also present the results of our XMM-Newtonoptical spectroscopy campaign of 3C 390.3 performed in 2004 October. An optical spectrum was acquired at Keck Observatory simultaneously to the XMM-Newton EPIC observations in order to enable us to compare the profiles of the $\mathrm{Fe} \mathrm{K} \alpha$ and doublepeaked Balmer lines, testing the idea that the former originates in the disk/BLR. While this paper focuses on a spectroscopic study, a detailed discussion of the X-ray timing properties of 3C 390.3 based on the various data sets can be found in a companion paper by Gliozzi et al. (2009).

The paper is organized as follows. After describing the source properties and previous observations in Section 2, in Section 3 the data reduction of the new observations is presented. We describe the spectral fits to the various data sets in Section 4. In Section 5, we discuss the multiwavelength spectral energy distribution (SED). Results are summarized in Section 6 and discussed in Section 7, with conclusions following in Section 8. Throughout this paper, a concordance cosmology with $H_{0}=$ $71 \mathrm{~km} \mathrm{~s}^{-1} \mathrm{Mpc}^{-1}, \Omega_{\Lambda}=0.73$, and $\Omega_{m}=0.27$ (Spergel et al. $2003)$ is adopted. The energy spectral index, $\alpha$, is defined such that $F_{v} \propto v^{-\alpha}$. The photon index is $\Gamma=\alpha+1$.

\section{PROPERTIES OF 3C 390.3}

The BLRG 3C 390.3, located at $z=0.056$, is well studied at all wavelengths from radio to X-rays. In the radio it is a classical double-lobed FRII radio galaxy (Pearson \& Readhead 1988) and the core exhibits superluminal motion (Alef et al. 1996). From the apparent velocity and the core dominance, a jet inclination angle with respect to the line of sight of $30^{\circ}<\theta<35^{\circ}$ was inferred (Giovannini et al. 2001).

We can assess the jet contribution to the nuclear emission by calculating the expected Doppler factor from the jet inclination angle, $30^{\circ}<\theta<35^{\circ}$, and jet velocity, $\beta \sim 0.96-0.99$ (Giovannini et al. 2001). This yields Lorentz factors $\Gamma_{L}$ in the range 3.6-7.1 and thus Doppler factors $\delta=0.6-1.7$. While the range of Doppler factors formally does not exclude jet amplification, we show in Section 7.3 by direct modeling of the SED that the beamed jet emission does not make a significant contribution to the optical-to-X-ray emission from the nucleus unless the accretion-flow emission is in a very low state, which was not the case for the observations presented here.

In the optical, 3C 390.3 is known for exhibiting doublepeaked optical emission lines (Eracleous \& Halpern 1994). By fitting the line profiles with a disk model, an inclination angle between the disk axis and the line of sight can be derived; for 3C 390.3 this gave $i=\left(26_{-2}^{+4}\right)^{\circ}$ (Eracleous \& Halpern 1994), very close to the range derived from the radio. Our optical spectrum simultaneous to XMM-Newton provides similar constraints (Section 4.5).

The source is also well studied in the X-ray band (Grandi et al. 1999, and references therein). At these wavelengths it is variable, showing large-amplitude variations at both soft and hard X-rays on timescales of weeks to months (Leighly \& O'Brien 1997; Gliozzi et al. 2003). It was observed with all previous X-ray observatories, including ASCA, which provided the first highquality X-ray spectrum (Eracleous et al. 1996). The ASCA $40 \mathrm{ks}$ spectrum showed a rather hard X-ray continuum with photon index $\Gamma=1.7$, and a resolved $\mathrm{Fe} \mathrm{K} \alpha$ emission line at $6 \mathrm{keV}$ with an equivalent width $\mathrm{EW} \sim 170 \mathrm{eV}$ and a Gaussian profile consistent with an origin from the outer $\left(r>250 r_{g}\right)$ regions of the disk.

A BeppoSAX observation of 3C 390.3 confirmed the presence of the Fe $\mathrm{K} \alpha$ line and showed a strong reflection bump, $R \sim 1$ (Grandi et al. 1999). 3C 390.3 was recently detected above $10 \mathrm{keV}$ with the BAT experiment onboard Swift (Tueller et al. 2008; see Section 3.4) and with International Gamma-Ray Astrophysics Laboratory (INTEGRAL; Bird et al. 2007). The BAT data are used in this paper and fitted jointly with $S u z a k u$.

The Galactic column density in the direction to 3C 390.3 is $3.5 \times 10^{20} \mathrm{~cm}^{-2}$ (Kalberla et al. 2005). Previous X-ray observations detected excess amount of cold absorption above this value (Eracleous et al. 1996; Sambruna et al. 1999). Comparing measurements obtained at various epochs, Grandi et al. (1999) showed that the column density $N_{\mathrm{H}}$ in $3 \mathrm{C} 390.3$ varies on timescale of years, but is not correlated to changes of the X-ray continuum flux. There was a claim for the presence of warm absorption in the form of an absorption edge at $0.65 \mathrm{keV}$ from a reanalysis of the ASCA data (Sambruna et al. 1999).

\section{NEW OBSERVATIONS}

The log of the X-ray observations conducted in pointed mode is reported in Table 1 .

\subsection{XMM-Newton}

We observed 3C 390.3 with XMM-Newton on 2004 October 8-9 and 17 for $50 \mathrm{ks}$ and $20 \mathrm{ks}$, respectively. All of the EPIC cameras (Strüder et al. 2001; Turner et al. 2001) were operated in small window mode to prevent photon pile-up, and with medium filters, due to the presence of bright nearby sources in the field of view. The recorded events were screened to remove known hot pixels and other data flagged as bad; only data with FLAG $=0$ were used. The data were processed using the latest CCD gain values. For the temporal and spectral analyses, events corresponding to pattern 0-12 (singles, 
Table 1

Journal of Pointed X-Ray Observations

\begin{tabular}{lcccccc}
\hline \hline Observatory & Date & Start Time (UT) & Exposure (ks) & Detector & Energy Range (keV) & Count Rate $\left(\right.$ counts s $\left.^{-1}\right)$ \\
\hline XMM-Newton & 2004 Oct 8-9 (A) & $19: 49$ & 32.6 & EPIC pn & $0.5-10$ & $14.66 \pm 0.02$ \\
& & & 45.4 & EPIC MOS & $0.6-10$ & $4.75 \pm 0.02$ \\
& & & 69.7 & RGS2 & $0.3-1.8$ & $0.601 \pm 0.003$ \\
XMM-Newton & \multirow{2}{*}{ 2004 Oct 17 (B) } & \multirow{2}{*}{$00: 12$} & 16 & EPIC pn & $0.5-10$ & $12.77 \pm 0.02$ \\
& & & 22.6 & EPIC MOS & $0.6-10$ & $4.14 \pm 0.01$ \\
Suzaku & & & 49.4 & RGS2 & $0.3-1.8$ & $0.507 \pm 0.003$ \\
& \multirow{2}{*}{ 2007 Dec 14-16 } & \multirow{2}{*}{$03: 25$} & 90 & XIS1 & $0.5-10$ & $2.548 \pm 0.005$ \\
& & & 85.4 & XIS0,3 & $0.5-10$ & $2.004 \pm 0.004$ \\
& & & 91.4 & HXD PIN & $10-30$ & $0.153 \pm 0.003$
\end{tabular}

Notes. Exposure after screening was applied to the data. The count rate is the net source intensity after screening and background subtraction.

doubles, triples, and quadruples) in the MOS cameras and 0-4 (singles and doubles only, since the pn pixels are larger) in the pn camera were accepted. Arf and rmf files were created with the XMM-Newton Science Analysis Software SAS v.7.0. Examination of the full-field light curves revealed the presence of one period of background flaring during the first observation. These events were excluded, reducing the effective exposure time to $33 \mathrm{ks}$ on October 8 . The net count rates are listed in Table 1 together with the effective exposures after data screening.

The source light curves and spectra were extracted from circular regions of radius $35^{\prime \prime}$ centered on the source. The background spectra and light curves were extracted from sourcefree circular regions on the same chip as the source and with extraction radius of $35^{\prime \prime}$ for the EPIC pn camera, and on a different chip with extraction radius of $70^{\prime \prime}$ for the EPIC MOS cameras. There are no signs of pile-up in the pn or MOS cameras according to the SAS task epatplot. The pn and MOS data were re-binned in order to contain 100 and 50 counts per channel, respectively, and fitted jointly. There is no flux variability within the single EPIC exposures; details of the timing analysis are given in a companion paper (Gliozzi et al. 2009).

The Reflection Grating Spectrometer (RGS) data for the two observations were reduced and analyzed separately and then compared. The data were reduced using the standard SAS task rgsproc and the most recent calibration files. The total exposure times are $\sim 69 \mathrm{ks}$ for the observation on October 8 and $\sim 49$ ks on October 17 for both RGS1 and RGS2. The RGS1 and RGS2 spectra were binned at the resolution of the instrument $(\Delta \lambda \sim 0.1 \AA)$. Thanks to the long exposure time and the brightness of the source we have high signal-to-noise ratio $(\mathrm{S} / \mathrm{N})$ in both of the RGS exposures: a total of $\sim 82,000$ and 49,000 net counts were collected in the two observations, respectively (Table 1).

\subsection{Suzaku XIS}

Suzaku observed 3C 390.3 on 2007 December 14-16 for a total exposure time of $100 \mathrm{ks}$. We used the cleaned event files obtained from version 2 of the Suzaku pipeline processing. Standard screening criteria were used, namely, only events outside the South Atlantic Anomaly (SAA) as well as with an Earth elevation angle $(\mathrm{ELV})>5^{\circ}$ were retained, and Earth daytime elevation angles (DYE_ELV) $>20^{\circ}$. Furthermore, data within $256 \mathrm{~s}$ of the SAA passage were excluded and a cutoff rigidity of $>6 \mathrm{GV}$ was adopted. The net exposure time after screening was $85 \mathrm{ks}$. The net source count rates are listed in Table 1.
A serendipitous source was detected in the XIS field of view with a very soft X-ray spectrum (see the Appendix). This source is likely to be a transient, as it is not present in any archival imaging X-ray observation of 3C 390.3.

The XIS spectra of 3C 390.3 were extracted from a circular region of 2.9 radius centered on the source. Background spectra were extracted from four circular regions offset from the main target and avoiding the serendipitous source and the calibration sources. The combined area of these four background regions is twice the area of the main target region. The XIS response (rmf) and ancillary response (arf) files were produced, using the latest calibration files available, with the ftools tasks xisrmfgen and xissimarfgen, respectively. The source spectra from the FI CCDs were summed, and fitted jointly with the BI (the XIS1) spectrum. The net XIS source spectra were binned with a minimum of 100 counts per bin.

\subsection{Suzaku HXD}

For the HXD-PIN data reduction and analysis we followed the latest Suzaku data reduction guide (the ABC guide ver. 2). ${ }^{14}$ We used the rev2 data, which include all four cluster units, and the best background available, which account for the instrumental background (Kokubun et al. 2007). ${ }^{15}$ The source and background spectra were extracted within the common good time interval and the source spectrum was corrected for the detector dead time. The net exposure time after screening was $90 \mathrm{ks}$.

The contribution of the diffuse cosmic X-ray background counts was simulated using the spectral form of Boldt (1987), assuming the response matrix for diffuse emission, and then added to the instrumental one. At the time of this analysis (2008 September), two instrumental background files are available; background A or "quick" background and background D or "tuned" background. We adopted the latter which is the latest release and which suffers lower systematic uncertainties of about $1.3 \%$, corresponding to about half uncertainty of the first release of the NXB. With this choice of background, 3C 390.3 is detected up to $70 \mathrm{keV}$ at a level of $23 \%$ above the background. The count rate in $10-30 \mathrm{keV}$ is $0.153 \pm 0.003$ counts s $\mathrm{s}^{-1}$. The HXD-PIN spectrum was binned in order to have an $\mathrm{S} / \mathrm{N}$ greater then 10 in each bin, and the latest response file released by the instrumental team was used. Because of its extremely soft X-ray spectrum (see the Appendix), the contribution of the XIS transient to the HXD flux is negligible.

\footnotetext{
$14 \mathrm{http} / / /$ heasarc.gsfc.nasa.gov/docs/suzaku/analysis/abc/

$15 \mathrm{ftp}: / /$ legacy.gsfc.nasa.gov/suzaku/doc/hxd/suzakumemo-2008-03.pdf
} 


\subsection{Swift BAT Observations}

The BAT spectrum was derived from the 9-months survey archive. The data reduction and extraction procedure of the eight-channel spectrum is described in Tueller et al. (2008). To fit the BAT spectrum, we used the latest calibration response diagonal.rsp and background files as of 2008 June. The source was detected in $15-100 \mathrm{keV}$ with a count rate $(1.64$ $\pm 0.07) \times 10^{-3}$ counts $\mathrm{s}^{-1}$. Inspection of the long-term online BAT light curve of 3C 390.3 shows that there is no variability on timescales of weeks or longer.

\subsection{Optical Spectroscopy}

An optical spectrum of 3 C 390.3 was obtained on the night of 2004 October 9 UT during the first XMM-Newton observation at the Keck II telescope using the Echellette Spectrograph and Imager (ESI; Sheinis et al. 2002). The galaxy was observed in ESI echelle mode, which covers 3800-11000 A over 10 echelle orders. The ESI exposure began at 5:26 UT with a duration of $600 \mathrm{~s}$. A 0.75 wide slit was used, giving an instrumental dispersion of $\sigma_{i} \approx 22 \mathrm{~km} \mathrm{~s}^{-1}$. The slit was oriented at the parallactic angle and the air mass was 2.05.

The data were reduced following standard techniques and the spectrum was extracted with an extraction width of $1^{\prime \prime}$. Wavelength calibration was done using exposures of internal $\mathrm{HgNe}, \mathrm{Xe}$, and $\mathrm{CuAr}$ comparison lamps. Flux calibration and correction for telluric absorption bands were done using an exposure of the standard star BD+284211, which was observed in evening twilight. Due to scattered clouds during the observation, as well as slit losses, the absolute flux scale of the spectrum is subject to substantial uncertainty. After flux calibration, the individual echelle orders were combined into a single spectrum with a uniform linear dispersion of $0.25 \AA$ pixel $^{-1}$.

\section{SPECTRAL ANALYSIS}

All spectral fits to the X-ray data were performed using XSPEC V.11.3.2ag, except for the fits with ionized reflection for which v.12.4.0 was used. The significance of adding free parameters to the model was evaluated with the $F$-test, with associated probability $\mathrm{P}_{F}$. All uncertainties quoted are $90 \%$ for one parameter of interest unless otherwise noted.

\subsection{The RGS Spectra}

For each epoch, the RGS1 and RGS2 data sets were fitted jointly with a single absorbed power-law model, keeping the absorption fixed to the Galactic value, in the energy range 0.35 $1.8 \mathrm{keV}$. This gave $\chi^{2}=559 / 458$ and 548/458 for observations A and B, respectively. Figures 1(a)-(b) show the RGS spectra and the residuals of the power-law and Galactic absorption model, stretched out in two panels to show the details. The best-fit parameters are $\Gamma=1.79 \pm 0.02$ for observation $\mathrm{A}$ and $\Gamma=1.77 \pm 0.02$ for observation $\mathrm{B}$; the observed fluxes are $F_{0.3-2 \mathrm{keV}}=2.5 \times 10^{-11} \mathrm{erg} \mathrm{cm}^{-2} \mathrm{~s}^{-1}$ and $F_{0.3-2 \mathrm{keV}}=$ $2.1 \times 10^{-11} \mathrm{erg} \mathrm{cm}^{-2} \mathrm{~s}^{-1}$, respectively.

Allowing the absorption to vary, we found that the fit improves by $\Delta \chi^{2} \sim 36$ and 50 in the two exposures yielding $N_{\mathrm{H}} \sim 6 \times 10^{20} \mathrm{~cm}^{-2}$, in slight excess over the Galactic value. Fixing the local absorption to the Galactic value and adding a rest-frame absorber, we find an intrinsic column density of $N_{\mathrm{H}}^{z}=(1.0 \pm 0.6) \times 10^{20} \mathrm{~cm}^{-2}$ and photon index is now $\Gamma=1.87 \pm 0.05$ for the October $8-9$ observation, and
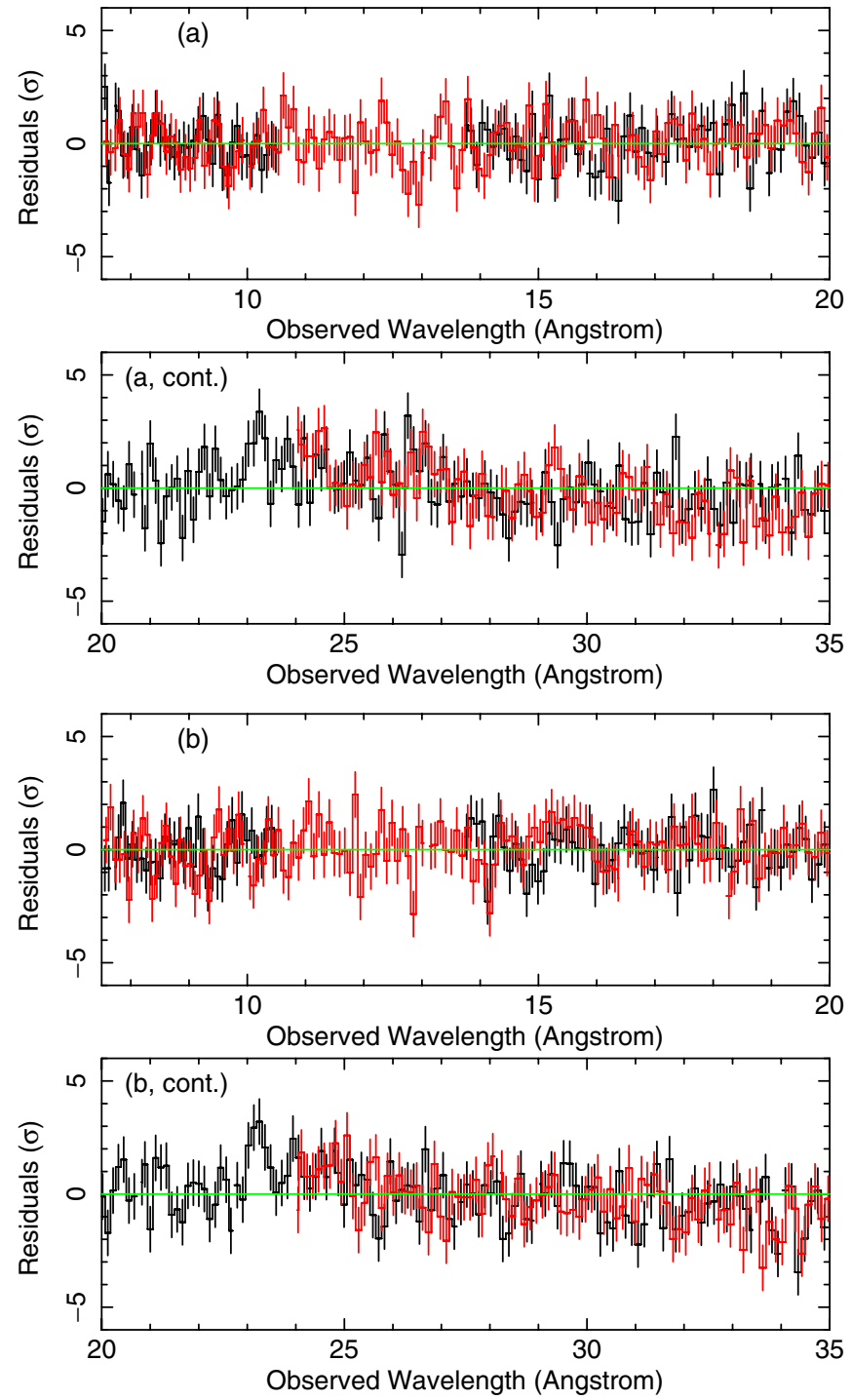

Figure 1. Plots of the XMM-Newton RGS spectra of 3C 390.3 from observations A ((a), top two panels) and B ((b), bottom two panels). The RGS1 data are plotted in black, while the RGS2 data are plotted in red. Shown are the residuals of a fit with a single power law and a column density fixed to the Galactic value. There is no evidence for discrete features in absorption. A significant narrow ( $\sigma_{L} \sim 5$ $\mathrm{eV}$ ) emission line is detected at $23.2 \AA$ with $\mathrm{EW} \sim 5 \mathrm{eV}$. The line energy centroid is coincident with the forbidden line of $\mathrm{O}$ VII at $0.564 \mathrm{keV}$ in the source's rest frame, which may arise from the NLR on kpc scales.

(A color version of this figure is available in the online journal.)

$N_{\mathrm{H}}^{z}=(2.0 \pm 0.7) \times 10^{20} \mathrm{~cm}^{-2}$ and $\Gamma=1.93 \pm 0.06$ for the October 17 observation.

Inspection of Figure 1 shows that there are no prominent spectral features in the RGS data, except for an emission line in the RGS1 data sets of both epochs at $\sim 23.5 \AA(0.56 \mathrm{keV})$. We fitted the RGS1 from both epochs A and B by adding a Gaussian line to the single power-law model; the RGS2 data were not included in this fit because the RGS2 CCD chip corresponding to the $\mathrm{O}$ viI triplet wavelength range malfunctioned shortly after launch in 1999 (Pollock 2008). The joint fit provides a rest-frame energy and flux for the Gaussian of $E_{l}=0.564 \pm 0.002 \mathrm{keV}$ and $F_{l}=(1.9 \pm 0.5) \times 10^{-4} \mathrm{ph} \mathrm{cm}^{-2} \mathrm{~s}^{-1}$ having fixed the line width to its best-fit value, $\sigma_{l}=5 \mathrm{eV}$; the line $\mathrm{EW}$ is $\sim 5 \mathrm{eV}$. The feature is significant, with $\Delta \chi^{2}=54$ for two additional degrees of freedom (dof) corresponding to $P_{F}>99 \%$. It can be identified with the forbidden $\mathrm{O}$ vII line from the distant 

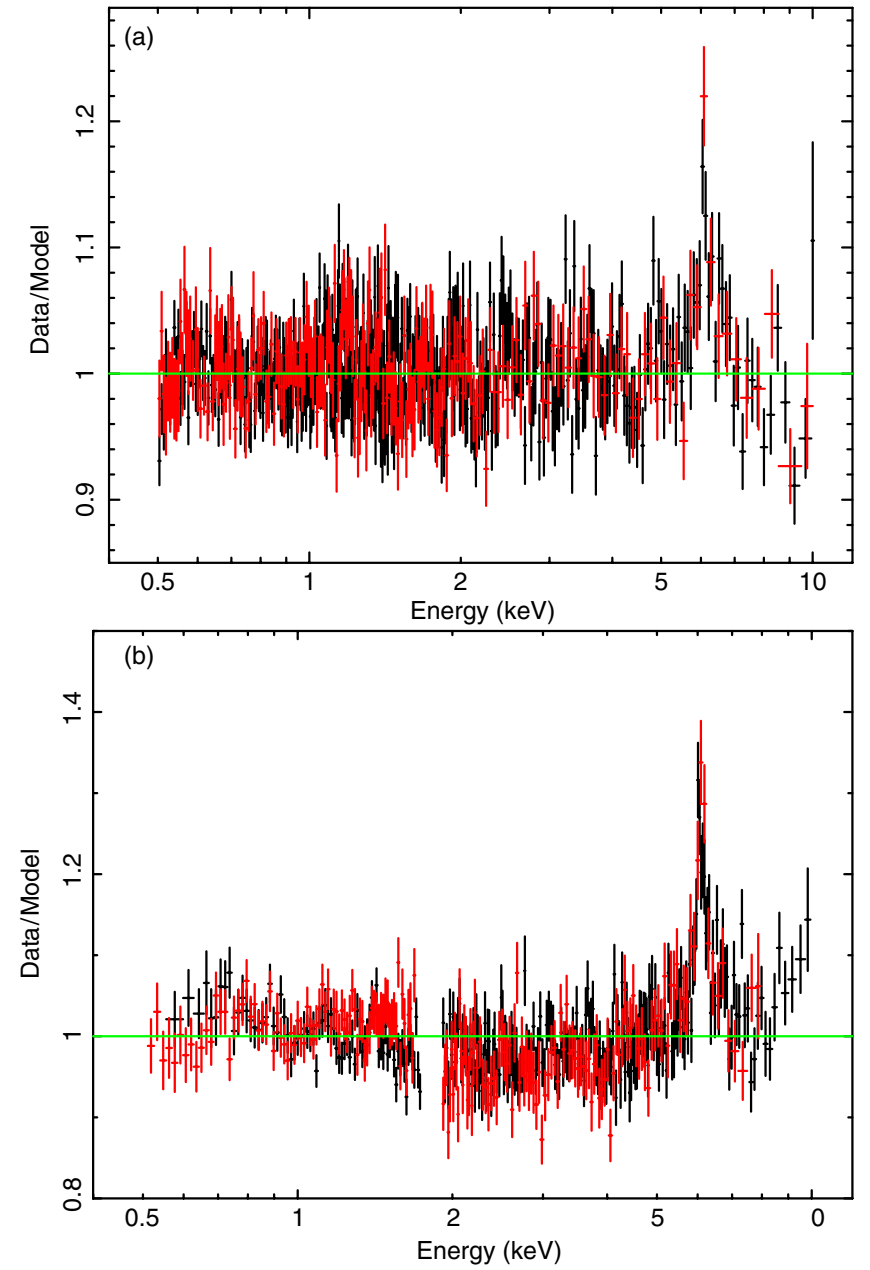

Figure 2. (a) Residuals of the EPIC pn spectra from observations A and B fitted jointly relative to a single power-law model. (b) Same for the Suzaku XIS data. The summed XIS03 data are shown in black, the XIS1 is in red. The data gap between 1.7 and $2.0 \mathrm{keV}$ is due to the detector $\mathrm{Si}$ edge, where the calibration is still not completely reliable.

(A color version of this figure is available in the online journal.)

narrow -line regions (NLR), as observed in Seyferts (Guainazzi et al. 2005). Although the line is highly statistically significant, it coincides with the observed wavelength of the neutral OI edge near $23 \AA$ (at $z=0$ ), which may add some systematic uncertainties to the measured value.

In short, our analysis of the high S/N RGS spectrum of 3C 390.3 shows that there is no evidence for discrete features in absorption in the energy range $0.35-1.8 \mathrm{keV}$. The data can be adequately fitted with a single power-law model with column density slightly in excess to the Galactic value. An emission line is found at $0.564 \mathrm{keV}$, and its proposed interpretation is [O VII] emission from the distant narrow-line region.

\subsection{The $0.5-10 \mathrm{keV}$ Continuum}

We first describe spectral fits to the XMM-Newton EPIC data focusing on the continuum in the energy range $0.5-10 \mathrm{keV}$. Previous spectral studies demonstrated that the broadband X-ray continuum of 3C 390.3 cannot be described by a simple power law, indicating the presence of variable cold absorption, an iron $\mathrm{K} \alpha$ line, and the onset of cold reflection (see Section 2). Figure 2(a) shows the residuals of the EPIC data fitted to a single power-law model (only the pn data for both observations are shown for clarity). Indeed, a single power law absorbed by Galactic $N_{\mathrm{H}}$ provides a poor fit to the EPIC spectrum of 3C 390.3, with $\chi^{2} /$ dof $=1590.1 / 1117$ and $\chi^{2} /$ dof $=956.9 /$ 758 for observations $\mathrm{A}$ and $\mathrm{B}$, respectively. Line-like residuals in the 5.5-7 keV energy range are present. A satisfactory fit is obtained for both XMM-Newton observations when a model including two power laws and a Gaussian line is used instead. The second power law is highly significant $\left(P_{F} \sim 99 \%\right)$ and parameterizes the onset of the reflection component above $8 \mathrm{keV}$, better visible in the Suzaku data (Figure 2(b)). When left free to vary, the absorbing column density is consistent with Galactic within large errors.

During observation A, the observed soft and hard X-ray fluxes are, respectively, $F_{0.5-2 \mathrm{keV}}=2.0 \times 10^{-11} \mathrm{erg} \mathrm{cm}^{-2} \mathrm{~s}^{-1}$ and $F_{2-10 \mathrm{keV}}=3.9 \times 10^{-11} \mathrm{erg} \mathrm{cm}^{-2} \mathrm{~s}^{-1}$, and the corresponding luminosities $L_{0.5-2 \mathrm{keV}}=2.0 \times 10^{44} \mathrm{erg} \mathrm{s}^{-1}$ and $L_{2-10 \mathrm{keV}}=$ $2.9 \times 10^{44} \mathrm{erg} \mathrm{s}^{-1}$. A modest decrease of $\sim 12 \%-13 \%$ in the flux and luminosity values is observed during observation $\mathrm{B}$ with no accompanying spectral changes (Gliozzi et al. 2009). Thus, the EPIC pn spectra from observations A and B were fitted jointly; the results are reported in Table 2(a).

In the joint EPIC fits if left free to vary the column density converges to a value in excess of Galactic. Thus, we added a second absorber to the model, $\mathrm{N}_{\mathrm{H}}^{z}$, leaving the column free to vary and fixing the absorber's redshift to be equal to that of the source. Table 2(a) shows that the excess column density, if intrinsic, amounts to $\Delta N_{\mathrm{H}} \sim 2 \times 10^{20} \mathrm{~cm}^{-2}$ at $90 \%$ confidence.

Next, we examined the Suzaku XIS data. Spectral fits were performed in the energy ranges $0.5-10 \mathrm{keV}$ where the calibration is best and the background negligible. We first fitted the data jointly with a single power law plus Galactic column density and found a poor fit, as expected. The residuals, shown in Figure 2(b), present large-amplitude variations in the Fe $\mathrm{K}$ line region, 5-7 keV, and a trend of rising continuum above these energies.

The XIS data were then fitted with the same model used for the XMM-Newton data, i.e., two power laws, where the second power law parameterizes the rising continuum at higher energies. The fit yields photon indices for the two power laws $\Gamma_{1}=2.3$ and $\Gamma_{2}=1.4$, respectively. Again, there is a modest excess column density which, if intrinsic, is around $\Delta N_{\mathrm{H}} \sim 3 \times 10^{20} \mathrm{~cm}^{-2}$, very similar to what measured by EPIC. The best-fit parameters are reported in Table 2(b). The observed fluxes are $F_{0.5-2 \mathrm{keV}} \sim 1.8 \times 10^{-11}$ and $F_{2-10 \mathrm{keV}} \sim 3.1 \times 10^{-11} \mathrm{erg} \mathrm{cm}^{-2} \mathrm{~s}^{-1}$. Thus, the flux during the Suzaku observation is consistent with the flux measured by $X M M$-Newton two years earlier.

Joint fits were also performed for the EPIC and XIS data, using only the first epoch for the XMM-Newton observations for simplicity. The results are shown in Table 2(c). This fit yielded improved constraints in the Fe $\mathrm{K}$ line region (Section 4.3).

From the fits to the medium X-ray energies, then, it appears that a slight excess column density is present in 3C 390.3 over the Galactic value, in agreement with earlier findings (Grandi et al. 1999). However, we shall see later (Section 4.4) that with a more detailed parameterization of the broadband continuum in the $0.5-100 \mathrm{keV}$ energy range, the slight absorption excess disappears. In its place, a high-energy cutoff at $160 \mathrm{keV}$ for the power-law continuum is requested to model the primary continuum (Section 4.4 and Figure 5).

\subsection{The Fe K Emission Region}

As apparent in Figures 2(a)-(b) a prominent emission line is present at $6 \mathrm{keV}$ in both the EPIC and XIS data sets, coincident 
Table 2

Best Fit to the EPIC and XIS Spectra ${ }^{a}$

\begin{tabular}{|c|c|c|c|c|c|c|c|}
\hline 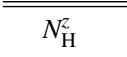 & 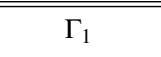 & 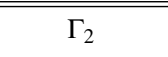 & 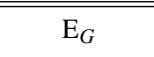 & $\sigma_{G}$ & $\overline{\mathrm{EW}_{G}}$ & $\overline{\chi^{2} / \mathrm{dof}}$ & $\mathrm{F}(2-10 \mathrm{keV})$ \\
\hline \multicolumn{8}{|c|}{ (a) EPIC pn + MOS $^{b}$} \\
\hline$\overline{2.0_{-0.7}^{+0.9}}$ & $2.09 \pm 0.12$ & $1.20 \pm 0.05$ & $\begin{array}{c}6.41 \pm 0.04 \\
6.56_{-0.45}^{+0.18}\end{array}$ & $\begin{array}{l}0.04_{-0.04}^{+0.10} \\
0.52_{-0.19}^{+0.09}\end{array}$ & $\begin{array}{l}26_{-12}^{+24} \\
85_{-27}^{+72}\end{array}$ & $2964 / 2733$ & 3.9 \\
\hline \multicolumn{8}{|c|}{ (b) XIS } \\
\hline $3.3 \pm 0.3$ & $2.32_{-0.04}^{+0.06}$ & $1.44 \pm 0.21$ & $\begin{array}{c}6.43 \pm 0.02 \\
6.33_{-0.5}^{+0.2}\end{array}$ & $\begin{array}{c}0.09 \pm 0.03 \\
0.61_{-0.23}^{+0.39}\end{array}$ & $\begin{array}{c}56 \pm 18 \\
79_{-27}^{+39}\end{array}$ & $1012 / 944$ & 3.1 \\
\hline \multicolumn{8}{|c|}{ (c) XIS + EPIC (A) } \\
\hline $2.0_{-0.9}^{+0.7}$ & $2.16 \pm 0.05$ & $1.34 \pm 0.11$ & $\begin{array}{c}6.40 \pm 0.02 \\
6.60_{-0.12}^{+0.36}\end{array}$ & $\begin{array}{c}0.07_{-0.04}^{+0.08} \\
0.40 \pm 0.30\end{array}$ & $\begin{array}{l}38_{-14}^{+38} \\
65_{-26}^{+23}\end{array}$ & $3062 / 2728$ & \\
\hline
\end{tabular}

Notes. Column 1: column density at redshift $z=0.056$, in $10^{20} \mathrm{~cm}^{-2}$; Columns 2 and 3: photon indices of the power-law components; Column 4: Gaussian line energy center in the source's rest frame, in keV; Column 5: Gaussian line width, in $\mathrm{keV}$; Column 6: observed EW calculated with respect to the total observed continuum, in $\mathrm{eV}$; Column 7: $\chi^{2}$ and degrees of freedom for the model; Column 8: observed flux in the energy range 2-10 keV in $\times 10^{-11} \mathrm{erg} \mathrm{cm}^{-2} \mathrm{~s}^{-1}$.

${ }^{\text {a }}$ The model consists of two power laws plus two Gaussians. A column density fixed to Galactic $\left(3.5 \times 10^{20} \mathrm{~cm}^{-2}\right)$ acts on all components.

b Observations A and B were fitted jointly (see Section 4.2).
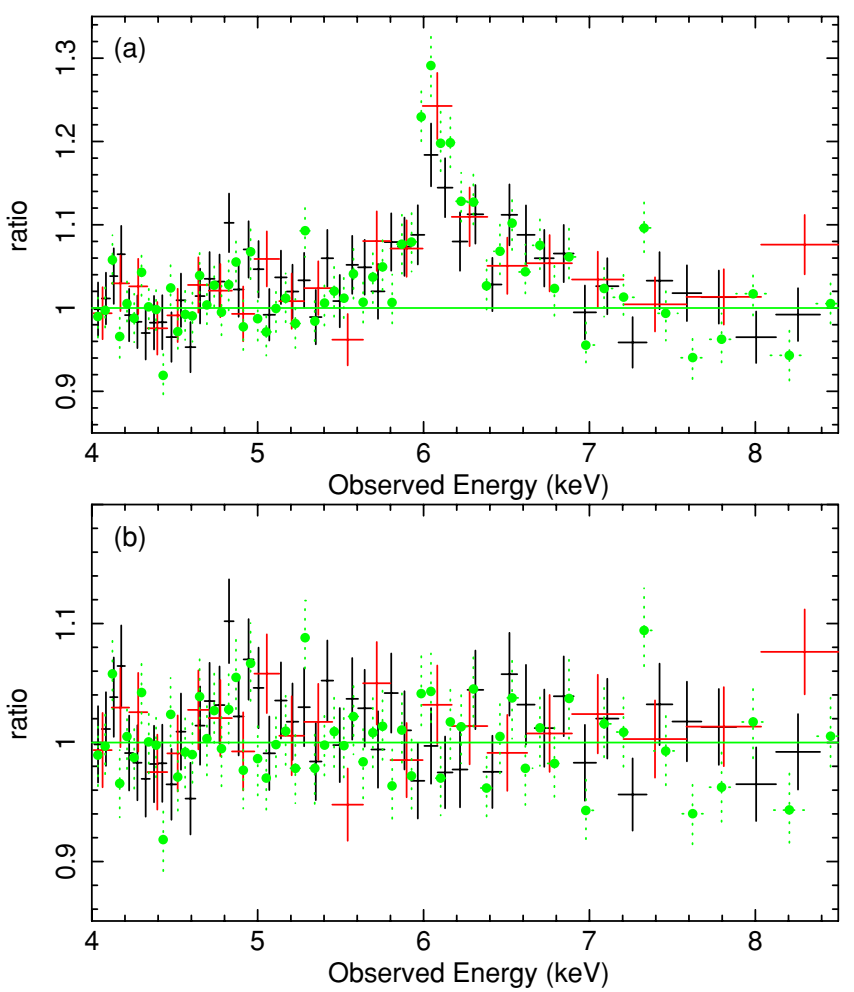

Figure 3. (a) Residuals of the fits to the XIS03 (green dots and dotted vertical lines) and EPIC (black and red) observations in the energy range of the $\mathrm{Fe} \mathrm{K} \alpha$ emission line with a single power-law model. A similar structure is seen at all epochs, consisting of a narrow component centered at an observed energy of $6 \mathrm{keV}$ and a broader bump between 5.8 and $7 \mathrm{keV}$. (b) Same as (a), but fitted with a power law plus two Gaussian lines (see Table 2(c)).

(A color version of this figure is available in the online journal.)

with the redshifted $\mathrm{Fe} \mathrm{K} \alpha$ line discovered with $A S C A$ (Eracleous et al. 1996). A more detailed view of the $\mathrm{Fe} \mathrm{K}$ region is presented in Figure 3(a), which shows a blow-up of the 4-9 keV region in the EPIC pn from the A (black) and B (red) data sets and from the XIS03 data (green). In all cases, the shape of the spectrum consists of a narrow line at an observed energy of $6 \mathrm{keV}$, easily identified with the $\mathrm{Fe} \mathrm{K} \alpha$ emission line at the rest energy of
$6.4 \mathrm{keV}$; and a weak, broad bump as a baseline between 5.5 and $7.5 \mathrm{keV}$. Thus, we added two Gaussian lines to the baseline continuum model in Table 2 to account for the narrow emission line at $6 \mathrm{keV}$ and the broad bump. The results are listed in Table 2 for the various data sets.

In the case of EPIC (Table 2(a)), the addition of the second broader Gaussian is significant at $99.7 \%\left(\Delta \chi^{2}=15\right.$ for 3 additional dof). Its centroid energy and width are $6.6 \mathrm{keV}$ and $0.5 \mathrm{keV}$, respectively. For the XIS data (Table 2(b)), the addition of the broader Gaussian improves the fit to the data by $\Delta \chi^{2}=$ 12, with $P_{F} \sim 99.4 \%$.

As apparent in Figure 3(a), despite the 26 months separating the two observations, the line profiles are very similar in both the XMM-Newton and Suzaku data sets. Thus, we performed joint fits to XIS and EPIC. The two data sets were fitted with a double power law plus two Gaussian lines; the bestfit parameters are reported in Table 2(c) and the residuals are shown in Figure 3(b). The cross-normalization factor between the XIS and PIN data was set at 1.09, as recommended for XIS nominal observations processed before 2008 July. ${ }^{16}$ Table 2(c) shows that the fit to the joint XIS and EPIC data set is good $\left(\chi^{2}=3062 / 2728\right)$. The $\mathrm{Fe} \mathrm{K} \alpha$ line at $6.4 \mathrm{keV}$ is narrow, with width $\sigma_{G}=0.07_{-0.04}^{+0.08} \mathrm{keV}$ and unresolved at $\gtrsim 96 \%$ confidence; its FWHM $=8800 \mathrm{~km} \mathrm{~s}^{-1}$ is consistent within a factor of 2 with the width of the Balmer emission lines (see Section 4.5). The line at $6.6 \mathrm{keV}$ has $\sigma_{G}=0.40 \pm 0.30 \mathrm{keV}$ or FWHM $=$ $43,000 \mathrm{~km} \mathrm{~s}^{-1}$, suggesting an origin from hot gas closer to the central engine. Specifically, the line energy is consistent with emission from $\mathrm{Fe} \mathrm{xxv} \mathrm{k} \alpha$.

In Table 2(c), both the narrow and the broad lines are highly significant, with $\Delta \chi^{2}=225$ and 27, respectively, corresponding to $P_{F}>99.999 \%$. In particular, the detection of the broad line is robust and independent of the parameterization of the underlying continuum. For example, fitting the $2-10 \mathrm{keV}$ continuum of 3C 390.3 with an absorbed power law plus a narrow Gaussian at $6 \mathrm{keV}$ yields a null intrinsic column density, but still requires a broad Gaussian line. Both narrow and broad lines are statistically required in all data sets, as summarized in Table 3, with their

\footnotetext{
16 ftp://legacy.gsfc.nasa.gov/suzaku/doc/xrt/suzakumemo-2007-11.pdf
} 
Table 3

Significance of the Narrow and Broad Fe K Lines

\begin{tabular}{lcc}
\hline \hline Data & Narrow & Broad \\
& $\Delta \chi^{2}\left(\mathrm{P}_{F}\right)$ & $\Delta \chi^{2}\left(P_{F}\right)$ \\
\hline EPIC & $89\left(4 \times 10^{-16}\right)$ & $15\left(2.7 \times 10^{-3}\right)$ \\
XIS & $138\left(8 \times 10^{-26}\right)$ & $12\left(5.7 \times 10^{-3}\right)$ \\
EPIC + XIS & $225\left(7 \times 10^{-45}\right)$ & $27\left(9.6 \times 10^{-5}\right)$
\end{tabular}

Notes. $\Delta \chi^{2}$ is the improvement in the fit when a Gaussian is added to the continuum model. $P_{F}$ is the corresponding probability according to the $F$-test.

significance increasing to $>99.999 \%$ in the joint fits compared to $99.5 \%$ for the two data sets separately.

We note that an intrinsically curved continuum with a cutoff around 3-4 keV, such as that expected from intrinsic cold absorption, is highly unlikely in this type-1 AGN. Moreover, there is no evidence from the longer wavelengths and no related absorption features were detected in the XIS in the Fe K region (Figure 3). This physical argument supports a line interpretation of the bump in 5-7 keV.

In summary, the XMM-Newton and Suzaku observations of 3C 390.3 confirm the presence of a narrow $\mathrm{Fe} \mathrm{K} \alpha$ emission line at $6.4 \mathrm{keV}$. Both data sets independently show, for the first time, that a broad bump dominates the 5-7 keV emission. The broad line has a rest-frame center energy of $6.6 \mathrm{keV}$, consistent with the $\mathrm{K} \alpha$ transition from He-like Fe (Fe XXv), thus suggesting the presence of highly ionized gas in the inner regions of 3 C 390.3 .

\subsection{The Spectrum at Energies $>10 \mathrm{keV}$}

The PIN spectrum from $15-70 \mathrm{keV}$ can be fitted well with a single power-law model with photon index $\Gamma=1.8 \pm 0.2$. The observed flux extrapolated to the $15-100 \mathrm{keV}$ band is $9.3 \times 10^{-11} \mathrm{erg} \mathrm{cm}^{-2} \mathrm{~s}^{-1}$.

A fit to the eight-channel BAT spectrum in the $15-100 \mathrm{keV}$ band with a single power-law model yields an acceptable fit $\left(\chi^{2}=1.5 / 5\right)$ with photon index $\Gamma_{\mathrm{BAT}}=2.0 \pm 0.1$ and a $15-$ $100 \mathrm{keV}$ flux of $8.2 \times 10^{-11} \mathrm{erg} \mathrm{cm}^{-2} \mathrm{~s}^{-1}$, within $12 \%$ of the PIN flux. Because of this and the slope similarity joint fits between the PIN and the BAT were justified. This is especially important in view of the extended sensitivity to higher energies provided by the BAT.

We performed joint fits to all the combined data sets: EPIC + $\mathrm{XIS}+\mathrm{PIN}+\mathrm{BAT}$, in the energy range $0.5-100 \mathrm{keV}$. Again, the XIS/PIN cross-normalization was fixed to 1.09 , while the one between Suzaku and BAT was left as a free parameter as the two observations are not simultaneous; the latter is consistent with one as indicated by the similar HXD and BAT fluxes. The data were fitted at first with a model consisting of a power law with a high-energy cutoff, plus two Gaussians to model the Fe $\mathrm{K} \alpha$ lines at 6.4 and $6.6 \mathrm{keV}$. Reflection off a cold slab (pexrav in XSPEC) was added as well, as this emission component was detected with BeppoSAX (Grandi et al. 1999) and is expected due to the presence of the $\mathrm{Fe} \mathrm{K} \alpha$ line. The cutoff energy of the power law was left as a free parameter, and its photon index was tied to the reflection components. The inclination angle of the disk was fixed to $26^{\circ}$ (Section 2); allowing it to vary in the range $25^{\circ}-35^{\circ}$, the total range derived from the radio and optical data, did not impact significantly the results. The Fe abundance was fixed to solar. The energy and width of the broad line were fixed at the values from the fits to the EPIC and XIS data in Table 2(c).
Table 4

Broadband Spectral Fits ${ }^{\mathrm{a}}$

\begin{tabular}{lcc}
\hline \hline Parameter & Best-Fit Value & Comment \\
\hline$\Gamma$ & (a) Power law + cold reflection + 2 Gaussians \\
\hline$E_{\text {cutoff }}$ & $1.72 \pm 0.02$ & \\
$R_{\text {cold }}$ & $161_{-62}^{+75}$ & Cutoff energy in keV \\
$E_{N}$ & $0.81 \pm 0.04$ & Cold reflection strength \\
$\sigma_{N}$ & $6.41 \pm 0.02$ & Narrow-line rest-frame energy in keV \\
$E_{N}$ & $0.03_{-0.03}^{+1.30}$ & Narrow-line width in keV \\
$E_{B}$ & $23_{-5}^{+7}$ & Narrow-line rest-frame equivalent width in eV \\
$\sigma_{B}$ & 6.6 & Broad-line rest-frame energy in keV, fixed \\
EW $_{B}$ & 0.40 & Broad-line width in keV, fixed \\
$\chi^{2} /$ dof & $55_{-20}^{+10}$ & Broad-line rest-frame equivalent width in eV \\
\hline
\end{tabular}

(b) Power law + cold reflection + Gaussian + ionized reflection + Kdblur

\begin{tabular}{lcc}
\hline$\Gamma$ & $1.59 \pm 0.03$ & \\
$E_{\text {cutoff }}$ & $157_{-47}^{+89}$ & Cutoff energy in keV \\
$R_{\text {cold }}$ & $0.49_{-0.14}^{+0.17}$ & Cold reflection strength \\
$E_{N}$ & $6.41 \pm 0.02$ & Narrow-line rest-frame energy in keV \\
$\sigma_{N}$ & $0.08 \pm 0.03$ & Narrow-line width in keV, fixed \\
$\mathrm{EW}_{N}$ & $40_{-9}^{+4}$ & Narrow-line rest-frame equivalent width in eV \\
$\xi$ & $2734_{-990}^{+975}$ & Ionization parameter \\
$r_{\text {in }}$ & $200(>20)$ & Inner disk radius in $r_{G}$ \\
$r_{\text {out }}$ & 1000 & Outer disk radius, fixed \\
$\log r$ & -3 & Emissivity profile, fixed \\
$\chi$ & $294 / 2552$ &
\end{tabular}

$\chi^{2} /$ dof $\quad 2941 / 2552$

$F(0.5-2 \mathrm{keV}) \quad 1.5 \times 10^{-11} \quad$ Observed total flux in erg $\mathrm{cm}^{-2} \mathrm{~s}^{-1}$

$F(2-10 \mathrm{keV}) \quad 3.8 \times 10^{-11} \quad$ Observed total flux in erg cm $\mathrm{cm}^{-2} \mathrm{~s}^{-1}$

$F(10-100 \mathrm{keV}) \quad 8.2 \times 10^{-11} \quad$ Observed total flux in erg cm $\mathrm{cm}^{-2} \mathrm{~s}^{-1}$

Note.

a Joint fits to the EPIC pn, XIS, PIN, and BAT data sets in 0.5-100 keV. A column density fixed to Galactic $\left(3.5 \times 10^{20} \mathrm{~cm}^{-2}\right)$ acts on all components.

The results of the fit with this model are reported in Table 4(a). We find that there is no need in this model for an excess absorption term: leaving the column density free to vary yields values consistent to the Galactic value. A possible explanation is that the apparent extra absorption column was mimicked by the subtle curvature of the continuum due to the high-energy cutoff, underlying the risks of narrowband X-ray modeling. The bestfit photon index is $\Gamma=1.72 \pm 0.02$, consistent with the value usually found for radio-loud AGN (e.g., Grandi et al. 2006). The data with the model, and the corresponding residuals, are shown in Figures 4(a)-(b). Figure 5 shows the confidence contours for the reflection strength, $R$, versus the cutoff energy, clearly demonstrating the need for the latter and the constraints on both. While formally the fit is acceptable, with a reduced $\chi_{r}^{2}=1.18$, Figure 4(b) shows nonunity residuals above $60 \mathrm{keV}$.

The presence of the $6.6 \mathrm{keV}$ line raises the interesting possibility that an ionized disk, and thus a continuum component from reflection from an ionized medium, may be present in 3C 390.3, as suggested earlier by Ballantyne et al. (2002). To test for this possibility, reflection from an ionized layer of gas (model reflion; Ross \& Fabian 2005) was explicitly included in the fit in lieu of the broad Gaussian line. The final model consists of a power law with high-energy cutoff, plus cold and ionized reflectors, plus a narrow Gaussian at $6.4 \mathrm{keV}$, plus a kdblur component to model smearing due to relativistic effects. The best-fit parameters are listed in Table 4(b). Again the value of the inclination angle has no impact on the model parameters and was fixed at $26^{\circ}$ in the fits. The shape of the ionized component around $6.6 \mathrm{keV}$ naturally accounts for the "bump" previously modeled with a broad Gaussian. We find 

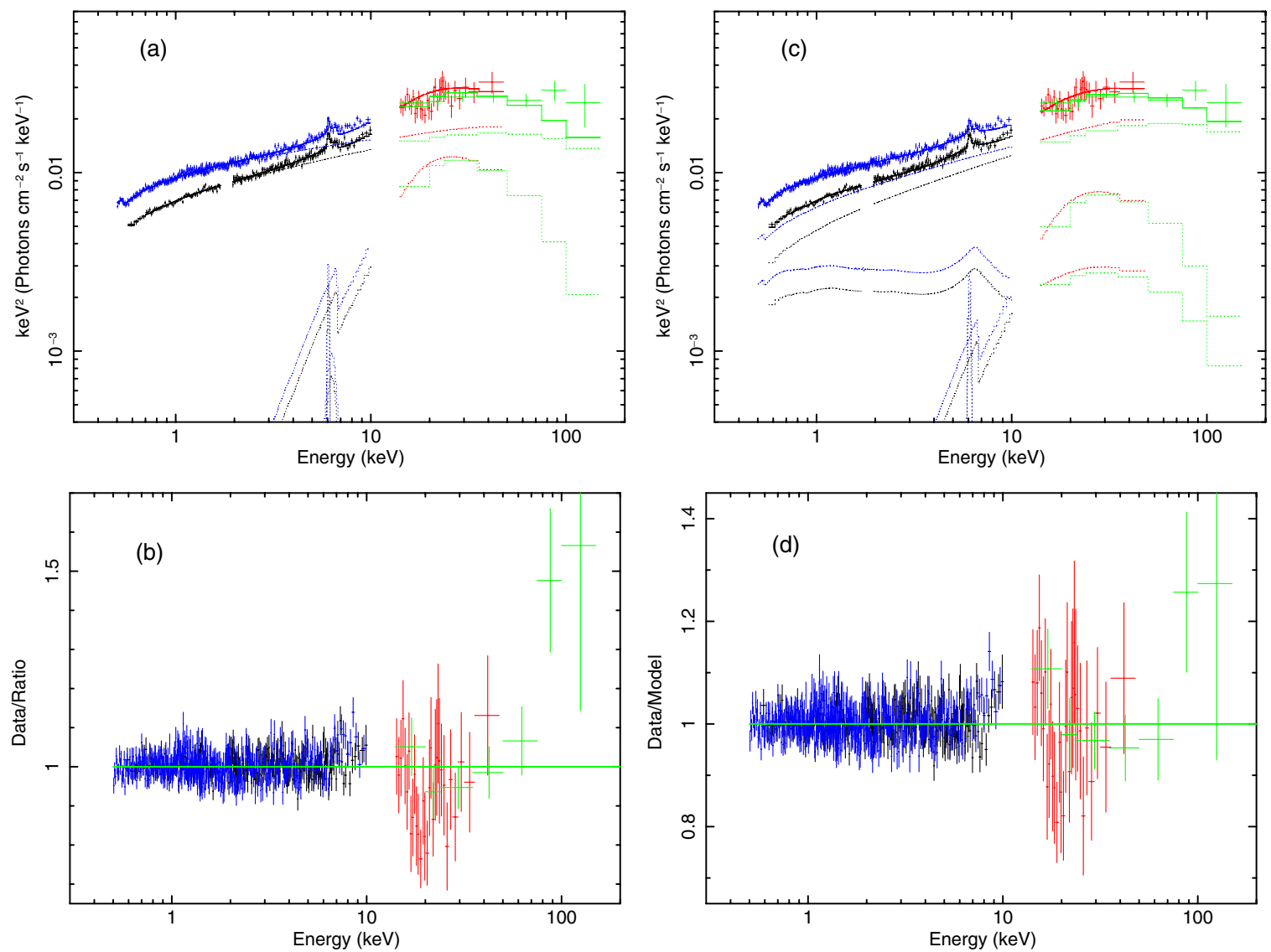

Figure 4. (a) Broadband EPIC, XIS, PIN, and BAT spectrum fitted with the model of Table 4(a), i.e., a power law with a cutoff plus cold reflection, a narrow Gaussian line at $6.4 \mathrm{keV}$, and a broad Gaussian centered at $6.6 \mathrm{keV}$. Only the pn observation A is shown for clarity. (b) Residuals of the model in (a). (c) The same data set as (a) but fitted with the model of Table 4(b), i.e., a cutoff power law plus cold reflection and a narrow Gaussian line at 6.4 keV, plus ionized reflection. (d) Residuals of the model in (c)

(A color version of this figure is available in the online journal.)

that both the cold and the ionized reflectors are required at high significance. Specifically, without the reflion component the fit with a cold reflector + Gaussian line gives a fit which is worse by $\Delta \chi^{2}=31$. The data and the model are shown in Figures 4(c)(d). The addition of the ionized reflector improves somewhat the residuals at the higher energies.

For the cold reflector, the albedo is $R=0.49_{-0.14}^{+0.17}$ and the narrow component of the line at $6.4 \mathrm{keV}$ has $\mathrm{EW}_{N}=40_{-9}^{+4} \mathrm{eV}$ against the total continuum. For an inclination of $26^{\circ}$ and the observed photon index of $\Gamma=1.6$, we expect $\mathrm{EW}_{N}=75 \mathrm{eV}$ for solar abundances (George \& Fabian 1991). Considering the uncertainties on the involved (fixed) parameters, such as, e.g., abundances, we can conclude that the narrow line is consistent with reflection off Compton-thick matter subtending a solid angle $R=0.5$ to the illuminating continuum, arising from reflection from the outer accretion disk/BLR. The cutoff energy of the power law is well determined at $157 \mathrm{keV}$, thanks to the addition of the BAT. The ionization parameter $\xi$ of the warm reflector is high, $\xi \sim 2700$, suggesting that $\mathrm{Fe}$ is mainly in He-like form, thus consistent with the results from the simple Gaussian parameterizations of the $\mathrm{Fe} \mathrm{K} \alpha$ line profile (Table 2(c)).

The ionized disk component contributes $20 \%$ of the total continuum flux above $10 \mathrm{keV}$, which is dominated by the cold component as observed in Figure 4(c). From this perspective, the ionized reflection strength is determined exclusively by the shape of the reflection bump around $6.6 \mathrm{keV}$. The latter sets

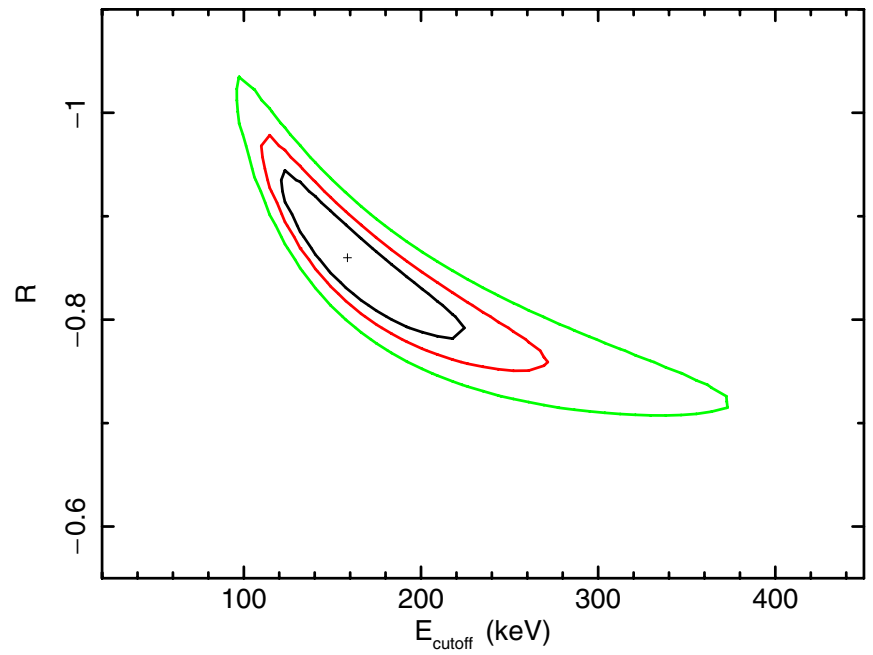

Figure 5. Plot of the cold reflection albedo, $R$, vs. the cutoff energy of the power law, from the fits to the broadband continuum with the model in Table 4(a). Contours are $68 \%, 90 \%$, and $99 \%$ confidence. A high-energy cutoff for the illuminating power law is needed and well constrained.

(A color version of this figure is available in the online journal.)

only marginal constraints on the inner disk radius. Assuming an emissivity profile proportional to $r^{-3}$, the $90 \%$ lower limit to the inner radius of the disk is $20 r_{g}$, for a fixed outer radius at $1000 r_{g}$ and an inclination angle of $26^{\circ}$. However, this result depends 


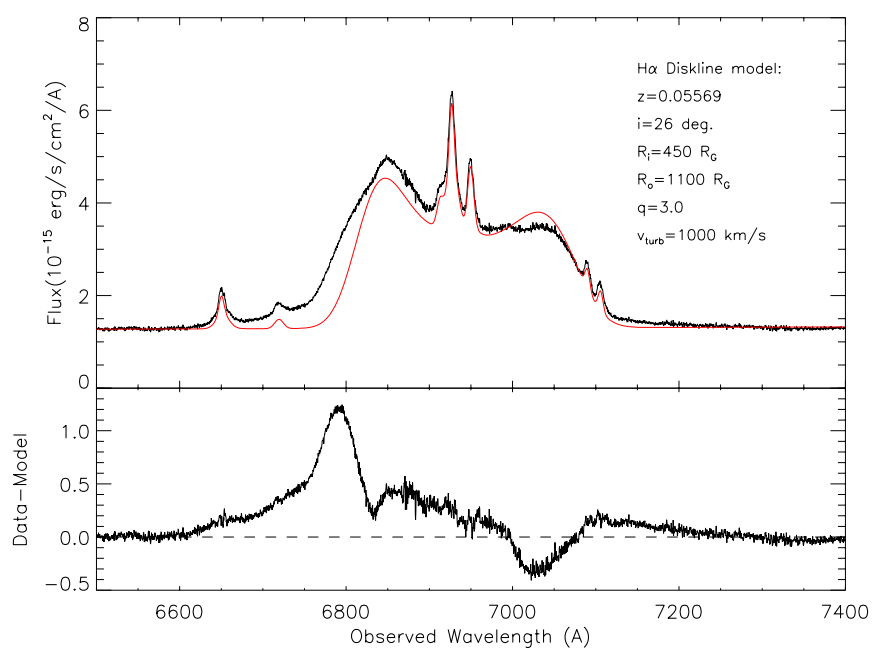

Figure 6. Zoom-in of the optical spectrum of 3C 390.3 around the $\mathrm{H} \alpha$ line. The spectrum was obtained at the Keck Observatory during the first XMM-Newton exposure (observation A in Table 1). The double-peaked $\mathrm{H} \alpha$ line is fitted (top panel) with a diskline model with the reported parameters. The bottom panel shows the residuals after subtracting the line best-fit model.

(A color version of this figure is available in the online journal.)

on the assumed disk emissivity law: with a flatter emissivity law, $\propto r^{-2}$, the limits on the inner radius are relaxed to smaller values $\left(2-3 r_{g}\right)$.

\subsection{The Optical Spectrum}

We fit the $\mathrm{H} \alpha$ region of the Keck ESI spectrum with the Interplanetary Monitoring Platform (IMP) spectral fitting code. The narrow [O I], [N II], [S II], and $\mathrm{H} \alpha$ lines were fitted by double Gaussian profiles with component widths in the range 170 $700 \mathrm{~km} \mathrm{~s}^{-1}$. We take the redshift of the $[\mathrm{S}$ II $]$ lines $(z=0.05569)$ as the galaxy rest frame for the purpose of fitting the broad $\mathrm{H} \alpha$.

The broad $\mathrm{H} \alpha$ was fitted with a diskline profile, convolved with a $1000 \mathrm{~km} \mathrm{~s}^{-1}$ turbulent velocity profile. The spectrum with the model is shown in Figure 6. The disk emissivity profile was assumed to follow $r^{-3}$, which corresponds to illumination by a central point source above the surface of the disk (see Section 4.4). The model which best fits the line width (FWHM $\sim 12,000 \mathrm{~km} \mathrm{~s}^{-1}$ ) and separation and relative strength of the blue and red peaks has an inclination of $i=26^{\circ}$ and inner and outer disk radii of 450 and $1100 r_{G}$, respectively. However, the observed broad $\mathrm{H} \alpha$ profile deviates from the model in two respects. As was noted by Eracleous \& Halpern (1994, and references therein), the ratio of blue/red peak flux is larger than predicted by any diskline model. This suggests asymmetric disk emission, such as might be produced by a spiral arm feature. It is worth noting that the blue excess in the profile is very similar to what was seen in another nearly contemporaneous observation, taken on 2004 September 21, by Gezari et al. (2007), while it was not present in other epoch spectra shown by these authors. Second, there is a very broad component (FWZI $\sim 4 \times 10^{4} \mathrm{~km} \mathrm{~s}^{-1}$ ) to the observed line, which could come from closer to the black hole, or perhaps scattering by hot electrons.

We then investigated whether or not the $\mathrm{H} \alpha$ profile is consistent with $\mathrm{Fe} \mathrm{K} \alpha$ line profile observed by XMM-Newton on the same night. We used XSPEC to fit the $2-10 \mathrm{keV}$ region of the EPIC pn data from observation A with a power-law continuum plus the best-fit $\mathrm{H} \alpha$ diskline model profile, only allowing the continuum and line normalization to vary. This produced a satisfactory fit to the EPIC continuum and neutral Fe $\mathrm{K} \alpha$ line, with $\chi^{2}=837$ for 791 dof. However, an equally good fit can be obtained with a simple Gaussian. We conclude that at the EPIC pn resolution, it is impossible to distinguish the double-peaked $\mathrm{H} \alpha$ profile from a Gaussian line of similar width. (We note that the XSPEC diskline profile does not include turbulent broadening, but this is insignificant compared to the instrumental broadening.)

Thus, an optical spectrum simultaneous to the first $X M M$ Newton observation confirms the presence of a double-peaked $\mathrm{H} \alpha$ line with a width FWHM $\sim 12,000 \mathrm{~km} \mathrm{~s}^{-1}$ and a profile consistent with disk emission from $450<r_{G}<1100$. The EPIC resolution is, however, insufficient to distinguish between a simple Gaussian profile and the $\mathrm{H} \alpha$ best-fit profile for the $\mathrm{Fe} \mathrm{K} \alpha$ line at $6.4 \mathrm{keV}$.

\section{THE BROADBAND SPECTRAL ENERGY DISTRIBUTION}

In order to define the SED of 3C 390.3, we compiled measurements from a variety of sources in the literature; the data and related references are listed in Table 5. We applied extinction corrections in the UV through IR bands using the extinction law of Seaton (1979) and adopting only a Galactic reddening of $E(B-V)=0.071$ (Schlegel et al. 1998). We also shifted the measurements to the rest frame of the source by applying the appropriate $K$-corrections. We also list in Table 5 two representative fluxes at $5 \mathrm{GHz}$ and $3 \mathrm{~mm}$. The former is a VLBI measurement which isolates emission from the radio core, while the latter is a measurement made with the IRAM $30 \mathrm{~m}$ single-dish telescope (it may include extended emission but we expect that at that frequency the core should dominate).

We plot the resulting SED in Figure 7 with red circles. For wavelengths shorter than $30 \mu \mathrm{m}$, we adopted as much as possible measurements through small apertures that isolate emission from the active nucleus. Cases where contamination from extended emission (from the host galaxy) is suspected, are identified in Table 5 and plotted as open circles in Figure 7. Our compilation of X-ray data is not complete, but it is enough to show the range of variability. We have included measurements since the early 1990s from ROSAT, ASCA, RXTE, and BeppoSAX, INTEGRAL, and from our present work. More extensive compilations of historical X-ray data can be found in Eracleous et al. (1996) and Grandi et al. (1999). Also plotted in Figure 7 are the contemporaneous multiwavelength data collected by Grandi et al. (1999) from radio to X-rays (green circles), together with the EGRET sensitivity limit (arrow) and the Fermi $5 \sigma$ detection curve for a one-year survey exposure.

The SED of the nuclear emission in Figure 7 shows two maxima in the optical-UV portion of the SED, and again at high energies above $10 \mathrm{keV}$ as implied by the hard X-ray continuum, which-as implied by our fits to the Suzaku + BAT data - cuts off around $160 \mathrm{keV}$ (Table 4). As discussed in Section 2 the jet is not expected to provide a significant contribution to the nuclear emission, unless the latter is very dim; we will return to this issue later. In Figure 7, the optical-to-hard X-ray emission is generally dominated by the accretion flow and the IR emission by the dusty torus.

There is obvious scatter in the SED in the optical and X-ray bands, which we attribute to intrinsic variability of the source. In Figure 7, we have plotted with vertical error bars the $\pm 1 \sigma$ variability amplitude at specific frequencies, as determined from intensive monitoring campaigns or from compilations of archival data (see details in Table 5). The observed variability amplitudes support the interpretation of the scatter as the result of variability. The X-ray measurements plotted in Figure 7 span 
Table 5

Spectral Energy Distribution of 3C 390.3

\begin{tabular}{|c|c|c|c|}
\hline $\log v_{\text {rest }}(\mathrm{Hz})$ & $\log L_{\text {rest }}\left(\mathrm{erg} \mathrm{s}^{-1}\right)$ & Note & Reference \\
\hline 9.72 & 41.11 & & 1 \\
\hline 11.02 & 42.31 & $\mathrm{a}$ & 2 \\
\hline 12.50 & 43.87 & & 3 \\
\hline 12.72 & 43.96 & & 3 \\
\hline 13.10 & 44.52 & $\mathrm{a}$ & 3 \\
\hline 13.32 & 43.92 & $\mathrm{a}$ & 4 \\
\hline 13.42 & 44.23 & $\mathrm{a}$ & 5 \\
\hline 13.42 & 44.41 & $\mathrm{a}$ & 3 \\
\hline 13.50 & 44.43 & & 3 \\
\hline 13.66 & 44.71 & $\mathrm{a}$ & 4 \\
\hline 13.97 & 44.04 & & 3 \\
\hline 14.16 & 43.65 & & 6 \\
\hline 14.16 & 43.96 & & 3 \\
\hline 14.16 & 44.06 & $\mathrm{a}$ & 7 \\
\hline 14.28 & 44.04 & $\mathrm{a}$ & 7 \\
\hline 14.30 & 43.87 & & 8 \\
\hline 14.42 & 44.08 & & 3 \\
\hline 14.46 & 44.10 & & 7 \\
\hline 14.55 & $43.77-43.85$ & $\mathrm{~b}$ & 9 \\
\hline 14.66 & $43.98-44.07$ & $\mathrm{~b}$ & 9 \\
\hline 14.76 & $43.95-44.09$ & $\mathrm{~b}$ & 9 \\
\hline 14.79 & $43.71-43.83$ & $\mathrm{~b}$ & 9 \\
\hline 14.79 & $43.37-43.64$ & $\mathrm{~b}$ & 10 \\
\hline 14.79 & 43.64 & & 11 \\
\hline 14.86 & $43.83-43.97$ & $\mathrm{~b}$ & 9 \\
\hline 15.14 & 44.06 & & 12 \\
\hline 15.16 & 43.44 & & 13 \\
\hline 15.18 & 43.65 & & 13 \\
\hline 15.23 & $43.19-43.72$ & $\mathrm{c}$ & 14 \\
\hline 15.23 & $43.53-43.95$ & $\mathrm{~b}$ & 15 \\
\hline 15.25 & 43.72 & & 13 \\
\hline 15.32 & 44.30 & & 12 \\
\hline 15.34 & 44.05 & & 13 \\
\hline 15.36 & $43.50-43.98$ & $\mathrm{~b}$ & 15 \\
\hline 15.37 & $43.24-43.77$ & $\mathrm{c}$ & 14 \\
\hline 17.41 & $43.83-44.10$ & $\mathrm{~b}$ & 16 \\
\hline $17.41,18.41$ & $43.91,44.23$ & $\mathrm{~d}$ & 17 \\
\hline $17.18,18.41$ & $43.63,44.00$ & $\mathrm{~d}$ & 18 \\
\hline $17.10,18.41$ & $43.62,44.01$ & $\mathrm{~d}$ & 16 \\
\hline $17.10,18.41$ & $43.93,44.16$ & d & 16 \\
\hline $17.10,18.41$ & $44.06,44.35$ & $\mathrm{~d}$ & 19 \\
\hline $16.88,18.23,19.41$ & $43.65,44.03,44.63$ & $\mathrm{~d}, \mathrm{e}$ & 20 \\
\hline $18.71,19.41$ & $44.31,44.56$ & $\mathrm{~d}$ & 21 \\
\hline $18.01,18.88,19.10$ & $44.02,44.28,44.42$ & $\mathrm{~d}, \mathrm{f}$ & 22 \\
\hline $17.10,18.41$ & $43.77,44.29$ & d & 23 \\
\hline $18.58,19.41$ & $44.36,44.69$ & d & 23 \\
\hline
\end{tabular}

Notes. (a) Contamination of the measured flux by extended emission from the host galaxy (or the large-scale radio jet and lobes in the case of the $3 \mathrm{~mm}$ measurement) is possible. (b) Range of luminosities indicating the $\pm 1 \sigma$ spread due to variability, as measured in an intensive monitoring campaign ranging from a few months to approximately a year. (c) Range of luminosities indicating the $\pm 1 \sigma$ spread due to variability, as measured from archival data spanning a few decades. (d) Groups of frequencies and luminosities define power law or broken power-law models that describe X-ray spectra. (e) Power-law model representing the INTEGRAL measurements made over a $\sim 1$-year survey. We have assumed a photon index of 1.65, which reproduces the observed flux in the $20-40$ and $40-100 \mathrm{keV}$ bands and also agrees with the photon index of the Swift/BAT spectrum presented in this paper. (f) Broken power-law fit to the time-averaged RXTE spectrum presented by Gliozzi et al. (2003). This spectrum was the result of a 2-month monitoring campaign, in which the X-ray flux of the source varied by a factor of 2.2 .

References. (1) Alef et al. 1988; (2) Steppe et al. 1998; (3) Miley et al. 1984; (4) Ogle et al. 2006; (5) Siebenmorgen et al. 2004; (6) Heckman et al. 1983; (7) Balzano \& Weedman 1981; (8) Madrid et al. 2006; (9) Dietrich et al. 1998; (10) Veilleux \& Zheng 1991; (11) Bentz et al. 2006; (12) Martin et al. 2005; (13) Zirbel \& Baum 1998; (14) Zheng 1996; (15) O'Brien et al. 1998; (16) Leighly et al. 1997; (17) Malaguti et al. 1994; (18) Eracleous et al. 1996; (19) Evans et al. 2006; (20) Bird et al. 2007; (21) Grandi et al. 1999; (22) Gliozzi et al. 2003; (23) this work.

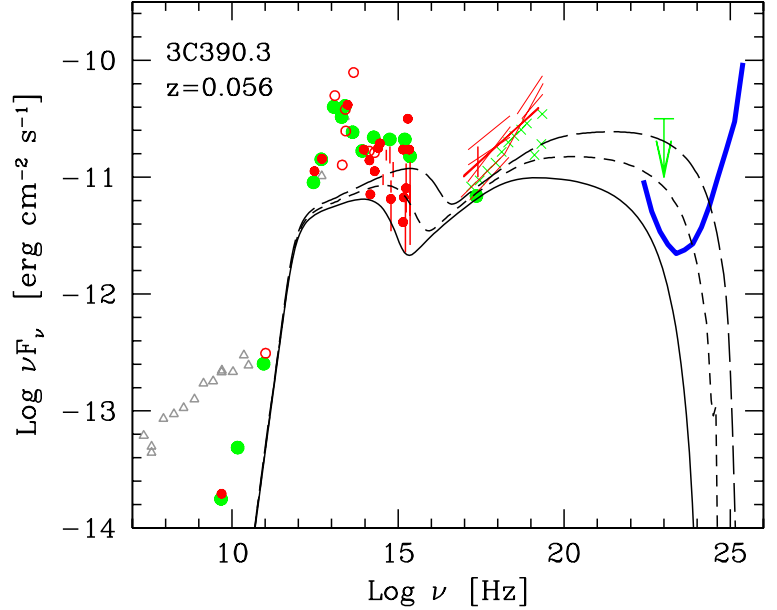

Figure 7. Spectral energy distribution of 3C 390.3, using the data in Table 5 (red circles) and the contemporaneous data from Grandi et al. (1999) and references therein (green circles). The gray triangles are from NED and represent the extended radio emission, included here for comparison. The open red dots in the optical-IR indicate suspected contamination from the host galaxy due to the large aperture. The blue curve at gamma rays is the one-year $(5 \sigma)$ sensitivity threshold of the LAT experiment on Fermi, while the arrow indicates the previous EGRET upper limit. A large spread of values is visible from IR to X-rays, due to the source's intrinsic variability. The continuous, dashed, and long-dashed lines are the predicted synchrotron self-Compton (SSC) emission from the jet assuming "typical" blazar parameters and for $\gamma_{\max }=1.5,3$, and $7 \times 10^{4}$, respectively, and beaming $\delta=1.75$, the maximum value for a jet inclination of $33^{\circ}$ (Section 7.3). The emission from 3C 390.3 is mostly due to accretionrelated processes. However, during low accretion states the jet can significantly contribute to the optical-to-X-ray continuum. The jet is expected to dominate at very high energies.

(A color version of this figure is available in the online journal.)

a factor of 3.5 in the $2-10 \mathrm{keV}$ luminosity. This range includes all the X-ray measurements in the compilations of Eracleous et al. (1996) and Grandi et al. (1999), save for the OSO7 observations from the early 1970s, which give a $2-10 \mathrm{keV}$ flux that is three times larger than the largest flux presented here, albeit with large error bars.

We determined upper and lower limits to the (variable) bolometric luminosity from $5 \mathrm{GHz}$ to $100 \mathrm{keV}$ by integrating the upper and lower envelopes of the SED. We find that the bolometric luminosity is in the range (1-4) $\times 10^{45} \mathrm{erg} \mathrm{s}^{-1}$. Combining this luminosity range with the black hole mass of $(5 \pm 1) \times 10^{8} M_{\odot}$ (Nelson et al. 2004), we infer an Eddington ratio in the range $0.01-0.07$, in agreement with the range $0.02-$ 0.04 obtained by Lewis \& Eracleous (2006).

\section{SUMMARY OF OBSERVATIONAL RESULTS}

The Fe K region. For the first time Suzaku and XMM-Newton independently provide evidence that the $5-7 \mathrm{keV}$ emission from 3C 390.3 is complex. Two components are required: a narrow line with $\mathrm{EW}_{N}=40 \mathrm{eV}$ and FWHM $=8800 \mathrm{~km} \mathrm{~s}^{-1}$ from "cold" Fe at $6.4 \mathrm{keV}$ and a broad bump centered at $6.6 \mathrm{keV}$ which suggests emission from He-like Fe. The $\mathrm{Fe} \mathrm{K} \alpha$ and the new $6.6 \mathrm{keV}$ emission component are very similar in shape in both the XMM-Newton and Suzaku data sets taken 26 months apart.

The X-ray continuum. The broadband $0.5-100 \mathrm{keV}$ continuum is well described by a power law with a photon index $\Gamma=1.6$, with a well defined cutoff energy at $160 \mathrm{keV}$. Two reflectors are necessary to interpret the high-energy spectrum of 3C 390.3, a cold one with $R=0.5$ and an ionized one, 
with ionization parameter $\xi \sim 2700$. The broader line profile is consistent with emission from regions of the disk outside $20 r_{g}$ from the central black hole, although this result depends on the assumed emissivity profile of the disk.

The soft X-ray spectrum. No features in absorption are detected in the very high $\mathrm{S} / \mathrm{N}$ RGS spectrum; a narrow $\left(\sigma_{L} \sim 5 \mathrm{eV}\right)$ emission line due to $\mathrm{O}$ VII is present at $0.564 \mathrm{keV}$, indicating a possible origin in the NLR.

The broadband SED. Large scatter is present in the optical$\mathrm{UV}$ and at medium-hard X-rays, due to intrinsic variability of the AGN. The Eddington ratio for 3C 390.3 is in the range 0.01-0.07.

\section{DISCUSSION}

\subsection{An Ionized Accretion Disk in 3C 390.3}

We presented new results from the analysis of our XMM-Newton and Suzaku observations of the BLRG 3C 390.3, obtained at two different epochs in 2004 and 2006, respectively. An integrated 9 month BAT exposure complemented the data set extending the energy coverage to $100 \mathrm{keV}$, and a Keck spectrum provided information on the optical emission simultaneously to $X M M$-Newton. From this rich suite of observations, a remarkably uniform picture of the inner regions of 3C 390.3 is starting to emerge.

In both the EPIC and the XIS spectra, the Fe K $\alpha$ emission line is narrow (by X-ray standards), with FWHM $\sim 8800 \mathrm{~km} \mathrm{~s}^{-1}$. Its width is consistent within a factor of 2 with the width of the broad $\mathrm{H} \alpha$ line measured simultaneously (Section 4.5), indicating that an origin of the $\mathrm{Fe} \mathrm{K} \alpha$ line from the BLR is likely. It has been argued (Eracleous \& Halpern 1994) that the broad Balmer lines originate from the accretion disk, consistent with their double-peaked profiles. Indeed, the BLR could form the outer part of the accretion disk (Eracleous \& Halpern 2003, and references therein). This material would also be responsible for the observed cold reflection of $R=0.5$. If the $\mathrm{Fe} \mathrm{K} \alpha$ line arises from the same parts of the disk responsible for the optical Balmer lines, a simple prediction is that its profile should be consistent with that of the observed $\mathrm{H} \alpha$ emission line. Unfortunately, as seen in Section 4.5, the EPIC (and indeed, XIS) resolution at $6 \mathrm{keV}$ is insufficient to distinguish a double-peaked profile from a simple Gaussian. Higher resolutions coupled with good sensitivity are required, a task for, e.g., the calorimeter on the upcoming Astro- $H$ mission.

Our new and most interesting result is the detection of a broad bump centered at a rest-frame energy of $6.6 \mathrm{keV}$, which we interpret as emission from He-like Fe. When fitted with a Gaussian it has an FWHM of $\sim 43,000 \mathrm{~km} \mathrm{~s}^{-1}$, indicating an origin from ionized matter relatively close to the central black hole. Modeling of the broadband continuum shows that this component arises from reflection from an ionized $(\xi \sim 2700)$ medium at $\gtrsim 20 r_{G}$ for an assumed emissivity profile, confirming the earlier suggestion by Ballantyne et al. (2002). We stress that the constraints on the inner radius are model dependent, and if a different emissivity profile is assumed the value of the inner radius is relaxed (see Section 4.4). On the other hand, an emissivity profile of $q=-3$ seems to be reasonable at least for Seyferts (e.g., Fabian et al. 2002). Evidence for a broad line due to H- or He-like Fe K emission was also claimed in the Suzaku observations of 3C 120 (Kataoka et al. 2007; see Section 7.2).

The intrinsic photon index we measure for 3C 390.3 after accounting for cold and warm reflection is $\Gamma=1.6$, close to the mean for radio-loud AGN (Grandi et al. 2006; Reeves \& Turner
2000). Previous X-ray monitoring of 3C 390.3 established that the photon index is anticorrelated with the flux on timescales of days or longer, i.e., the spectrum softens when the source brightens, as in nonjetted AGN (Gliozzi et al. 2009, 2003). Together with the constraints on the high-energy cutoff of the primary power law, this implies that thermal Comptonization dominates the emission below $100 \mathrm{keV}$ in $3 \mathrm{C} 390.3$, supporting the idea that the bulk of the X-ray continuum does not originate in a jet. However, a beamed nonthermal component, if present at all, is expected to contribute at much higher energies, or when the accretion-related emission is very faint (Section 7.3).

Another interesting result from the fits with the ionized disk model is that the inner regions of the disk do not seem to contribute to the $\mathrm{Fe} \mathrm{K}$ emission, as suggested by the shape of the broad $\mathrm{Fe} \mathrm{K} \alpha$ line at $6.6 \mathrm{keV}$, provided an emissivity profile $\propto r^{-3}$ is assumed for the disk. This seems to point to a structure that obscures or suppresses the emission from regions closest to the black hole. Since this feature may be present in at least another broad-lined radio-loud source observed with Suzaku (see below), let us discuss what plausible physical scenarios could account for it. There are three possible situations: (1) a highly ionized ion torus/ADAF occupying the inner disk, (2) obscuration by the base of a jet, or (3) lack of illumination. We review them in turn.

1. An ion torus/ADAF. In an ADAF, the central regions of the accretion disk are inflated into a hot $\left(T \sim 10^{12} \mathrm{k}\right)$, advectiondominated and thus radiatively inefficient ion torus. Most of the observed radiation would thus come from cooler $\left(T \sim 10^{9} \mathrm{k}\right)$ electrons in the outer parts of the disk via synchrotron (radio to IR) and inverse Compton (optical to X-rays). The reflection features would be weaker than in standard optically thick, geometrically thin disks because of the smaller solid angle subtended by the reprocessor (the disk) to the illuminating source in the ion torus solution.

Is an ADAF solution viable for 3C 390.3? The ADAF mode of accretion becomes important when the accretion rate decreases below a critical value, which is a few percent of the Eddington value, $0.01-0.1 M_{\text {Edd }}$ (assuming standard viscosity disk parameters; Narayan 2005). In Section 5, we inferred an Eddington ratio $0.01-0.07$ for $3 \mathrm{C} 390.3$, which is close to the limit for an ADAF. In LINERs and other low-luminosity AGNs where radiatively inefficient accretion flows are claimed to be present, the Eddington ratios are several orders of magnitude lower, $10^{-4}-10^{-6}$ (Ho 1999), placing 3C 390.3 on the boundary of ADAF-forming systems (Narayan 2005).

Another observational feature of ADAF-dominated systems is a steep optical-to-UV slope, and a relatively large X-rayto-UV flux ratio. For example, from his compilation of SEDs for seven low-luminosity AGNs, Ho (1999) derives an average optical-to-UV index $\sim 1.8$ with a range 1.0-3.1, compared to $0.5-1.0$ for classical bright AGN. The average optical-to-X-ray index, $\alpha_{\text {ox }}$, defined between $2500 \AA$ and $2 \mathrm{keV}$, is $\left\langle\alpha_{\text {ox }}\right\rangle=0.9$ versus 1.2-1.4 for luminous Seyferts (Ho 1999). Among the data plotted in Figure 7, some were collected sufficiently close in time to allow intraband comparison (Grandi et al. 1999; plotted as green dots). Focusing on these data points, it is apparent that significant UV emission relative to the optical and Xrays is indeed present. After correcting for reddening, these measurements yield an optical-to-UV slope $\sim 1$ and $\alpha_{\text {ox }} \sim 1.2$. Thus, the values of the optical-to-UV and optical-to-X-ray continuum in 3C 390.3 are closer to classical Seyferts than to low-luminosity ADAF candidates. Moreover, $\alpha_{\mathrm{ox}} \sim 1.2$ is completely consistent with the value found for classical, 
optically selected AGN with UV luminosity similar to 3C 390.3 (Steffen et al. 2006). We conclude that the optical-to-X-ray emission from 3C 390.3 is due to a standard accretion flow. The observed variability timescale of the optical-UV flux, of the order of weeks to months (O'Brien et al. 1998, and references therein), indicates an origin of the optical-UV light outside $100 r_{g}$.

We calculated the expected emission of a "pure" ADAF for a black hole of mass $5 \times 10^{8} M_{\odot}$ and accretion rate $0.01 M_{\odot} \mathrm{yr}^{-1}$, and standard accretion disk parameters as in the model of Narayan et al. (1998). We find that the expected ADAF contribution, dominated by bremmstrahlung emission peaking at $100 \mathrm{keV}$, is more than 2 orders of magnitude lower than the observed emission and the predicted jet emission (see Section 7.3). We thus conclude that, if there is an ADAF in 3C 390.3, its contribution to the observed broadband continuum emission is completely negligible. The observed optical-UV emission in this case would still be originating in the outer region of a standard accretion disk. Thus, whether there is an ADAF or not in 3C 390.3 is irrelevant from the point of view of its observed radiation.

2. Obscuration by the base of the jet. A second possibility is that the base of the jet accounts for the obscuration of the inner disk regions in 3C 390.3. Some direct observational evidence exists that this scenario is a plausible one, at least in an FRI radio galaxy. High-resolution radio observations of M87 show that the jet is still broad in the inner $30-100 r_{G}$, with an opening angle of $\sim 60^{\circ}$, with the collimation process still ongoing up to $1000 r_{G}$ (Junor et al. 1999). On the other hand, there is also evidence that the sub-pc radio jets of low- and high-power sources exhibit proper motions indicative of similar Lorentz factors in the range 3-10 (Giovannini et al. 2001). Thus, it is entirely possible that the phenomenology observed in M87 on scales less than $100 r_{G}$, i.e., a broad jet, is present also in FRII sources like 3C 390.3.

On these scales, thus, the jet particles are still relatively cold, i.e., nonrelativistic. On the other hand, we also know from the study of gamma-ray blazars that the jet becomes dissipative only after $100 r_{G}$ (Ghisellini \& Madau 1996). In order to absorb the radiation from the inner disk, the jet must be opaque for Thomson scattering, that is, $\tau_{T}=\sigma_{T} n_{e} r>1$. Since we are observing the jet at $\sim 30^{\circ}$ from its axis, we can assume that the absorbing region of the jet has a radius comparable to the inner emitting region of the disk, $r \sim 10 r_{g} \sim 10^{15} \mathrm{~cm}$, assuming $M_{\mathrm{BH}}=5 \times 10^{8} M_{\odot}$.

The electron density $n_{e}$ can be estimated assuming that the jet carries a kinetic power $L_{k}=\pi r^{2} n_{p} m_{p} \Gamma_{L}^{2} c^{3}$, where for simplicity we assume $n_{e}=n_{p}$, with $n_{p}$ the proton density. Therefore,

$$
n_{e}=\frac{L_{k}}{\pi r^{2} m_{p} \Gamma_{L}^{2} c^{3}}=2 \times 10^{8} \frac{L_{k, 47}}{r_{15}^{2} \Gamma_{L, 2}^{2}} \mathrm{~cm}^{-3},
$$

where we have assumed a kinetic power $L_{k, 47}$ (suitable for powerful FRII sources; e.g., Tavecchio et al. 2000) and the small $\Gamma_{L}=2$ is motivated by the assumption that the jet is still in the acceleration phase at these short distances. Thus, $\tau_{T} \sim 0.13$. Note, however, that this estimate is affected by large uncertainties. For example, the jet composition is unknown; assuming a pair-rich jet, $n_{e}>n_{p}$, would yield only a lower limit, $\tau_{T}>0.13$. Thus, we cannot exclude that under some conditions the jet base could provide a source of opacity via Thomson scattering to the X-ray emission from the disk.

3. Lack of illumination. Another plausible scenario is that the inner regions of the disk are not illuminated effectively. Lack of illumination could be achieved if, for example, the inner jet is narrowly collimated very close to its base and channels energy and radiation away from the disk surface. A similar scenario was recently proposed for the broad-line radio-loud source 4C +74.26 , where Suzaku observations also indicate that the $\mathrm{X}$-ray emission originate from the outer regions of the disk (Larsson et al. 2008). In principle, this scenario could be tested by future ALMA observations at high radio resolution mapping directly the inner jet.

The "aborted jet" model of Ghisellini et al. (2004) provides another possible framework. In this model, the disk in radioquiet Seyfert galaxies is illuminated not by a corona extended over the disk but by the heat generated in collisions by blobs of plasma moving at velocities smaller than the escape velocity in an aborted jet. The distance of the collisions as well as the amount of dissipation vary, such that if highly dissipative collisions occur close to the black hole, where gravitational bending is also at play, the disk is strongly illuminated yielding broad Fe K $\alpha$ lines with larger EW, as observed in Seyferts. In this scenario, one could hypothesize that in radio-loud sources like 3C 390.3 due to the larger blob ejection velocities the collisions are less efficient/frequent and they occur at higher distances from the black hole, effectively illuminating only the regions of the disk at some distance from it. This would yield weaker reflection features emerging from some distance from the central black hole.

\subsection{Comparison to Other BLRGs and to Seyferts}

Although only two other BLRGs have published Suzaku observations to-date, it is interesting to compare our results to those in the literature. A $160 \mathrm{ks}$ GTO observation of 3C 120 was presented by Kataoka et al. (2007). They found that the emission at $>2 \mathrm{keV}$ is dominated by the disk, namely, a power law with $\Gamma=1.7$, a cold reflector with $R=0.6-0.8$, and a narrow $\mathrm{Fe} \mathrm{K} \alpha$ line with FWHM $\sim 7500 \mathrm{~km} \mathrm{~s}^{-1}$ and $\mathrm{EW}=60 \mathrm{eV}$; interestingly, a weak broad component to the line is also required by the XIS data, which is interpreted as emission of $\mathrm{H}$ - or He-like Fe. If neutral $\mathrm{Fe}$ is assumed instead, the inclination angle is too large and inconsistent with the limits from the radio, $1^{\circ}<i<14^{\circ}$. Below $2 \mathrm{keV}$, the XIS spectrum is variable and best described by a soft $\Gamma \sim 2$ power-law emission, which Kataoka et al. attribute to synchrotron emission from the jet.

The radio-loud quasar $4 \mathrm{C}+74.26$ was also observed with Suzaku (Larsson et al. 2008). Similarly to 3C 390.3 and 3C 120, a narrow $\mathrm{Fe} \mathrm{K} \alpha$ line with $\mathrm{EW}=86 \mathrm{eV}$ is present in $4 \mathrm{C}+74.26$ together with cold reflection in the range $R=0.3-0.7$ and a $\Gamma=1.8$ continuum. The profile of the $\mathrm{Fe} \mathrm{K} \alpha$ line is consistent with emission from $\gtrsim 50 r_{g}$ from the central black hole, although a relativistic profile is not formally ruled out.

Thus, the currently published Suzaku observations of BLRGs suggest a broadly uniform picture where the continuum above $2 \mathrm{keV}$ in these sources is dominated by an accretion flow. As for the physical conditions of the accretion disk, in at least two sources-3C 120 and 3C 390.3-the evidence points toward relatively high ionization, and in two other sources (3C 390.3, $4 \mathrm{C}+74.26$ ) possibly to something obscuring the innermost regions or suppressing their emission. It is fair to say that the advent of Suzaku has finally provided the necessary sensitivity around the Fe K region to crack open the mystery of their central engine.

We can also attempt a preliminary comparison with the results for radio-quiet AGN. Recent, high $\mathrm{S} / \mathrm{N}$ observations of radioquiet Seyfert galaxies with XMM-Newton and Suzaku have 
revealed a significant diversity in their intrinsic properties. In the sample of 26 Seyferts studied with XMM-Newton EPIC by Nandra et al. (2007), the fraction of sources with a broad component to the $\mathrm{Fe} \mathrm{K} \alpha$ line is $70 \%$, of which only $45 \%$ require a relativistic profile with emission from a characteristic radius of $15 r_{G}$. Fits to the $\mathrm{Fe} \mathrm{K}$ line region with a model including reflection from the disk and a distant torus provide mean albedos $R=0.54$ for the disk and 0.45 for the torus, for a total $R \sim 1$. Taking into account the respective dispersions, the $1 \sigma$ range for Seyferts is $R=0.63-1.35$. The average photon index for the Nandra et al. sample is 1.86 with a dispersion of 0.22 , and again the $1 \sigma$ range is $1.64-2.08$. These results are being confirmed by the emerging Suzaku observations of Seyferts, which also show a significant spread of intrinsic photon indices, reflections strengths, and lack of broad components to the Fe $\mathrm{K}$ line in several cases (e.g., Turner et al. 2009; Ponti et al. 2009; Reynolds et al. 2009). In other Seyferts (e.g., MCG-5-23-16, Reeves et al. 2007; MCG-6-30-15, Miniutti et al 2007), the Fe K $\alpha$ line is still broad and has a relativistic profile. In general, however, it seems that in Seyferts the range of Fe K line widths and $R$ values is larger than previously thought-unresolved to relativistic for the former, negligible to $>1$ for the latter.

From this revised perspective, the distinction between BLRGs and Seyferts appears more blurred than in the ASCA and RXTE era. It is reasonable to state at this time that BLRGs may be clustered at one end of the distribution of X-ray spectral parameters for Seyferts, with significant overlap. This indicates that their central engines differ from most, but not all, radioquiet sources. Also interestingly, it implies that there may be a continuum of accretion properties across the two subclasses. The fundamental question still remains open, however: why are BLRGs capable of forming powerful jets while those Seyferts with similar accretion properties do not? Taken at face value our results reinforce the idea that conditions other than, or in addition to, accretion are required to form and launch a relativistic jet, e.g., a rapidly spinning black hole. As the Suzaku archive for both radio-loud and radio-quiet sources continues to grow, it will be interesting to explore further the relative distribution of observed X-ray parameters for the two classes.

Perhaps the cleanest distinction between BLRGs and Seyferts so far, aside from radio jets, is at soft X-rays. In this band, Seyferts often exhibit prominent spectral features in emission or absorption-signature of warm circumnuclear gas on pc scales-which so far have been scarce or absent in BLRGs. Our analysis of the XIS and RGS spectra in this paper reinforces this statement for 3C 390.3 (e.g., Figure 1). When the nuclear continuum is heavily obscured, as in the peculiar case of the BLRG 3C 445, soft X-ray emission lines from a photoionized nuclear medium can be detected (Sambruna et al. 2007; Grandi et al. 2007), a testament to the presence of gas in the inner regions of at least some sources. However, why BLRGs display no features in absorption at soft X-rays remains a puzzle. The only possible exception so far is the luminous BLRG/quasar 4C +74.26 (Ballantyne 2005, and references therein); here an XMM-Newton spectrum shows absorption edges of $\mathrm{O}$ VII and $\mathrm{O}$ VIII indicating photoionized gas with column densities $\approx 10^{21} \mathrm{~cm}^{-2}$ and $\log \xi \sim 60$ (Ballantyne 2005), at the lower end still of the distributions for Seyferts (e.g., George et al. 1998).

It must be noted, however, that very few bright BLRGs have high-quality soft X-ray spectra available at this time. Indeed, from an observational point of view, the study of BLRGs is plagued by the scarcity of available sources where to search for ionized absorption. Only four to five "famous" objects have been observed so far, because they are the only ones bright enough for the limited sensitivity of past X-ray detectors-with two of them, 3C 120 and 3C 111, located at low Galactic latitudes.

If confirmed by future observations of additional sources, the weakness or lack of absorption features at soft X-rays in BLRGs would qualify as (one of) the major observational distinctions between these sources and their radio-quiet sisters. From this perspective, it is intriguing and perhaps related that BLRGs have collimated jets, while Seyferts have winds. Is it possible that jet formation inhibits the production of an accretion disk wind, and thus of a warm absorber, and vice versa?

Helpful hints are provided by stellar-mass black holes with relativistic jets. A recent analysis of several epochs of Chandra HETGS and RXTE observations of the Galactic microquasar GRS 1915+105 (Neilsen \& Lee 2009) showed that the X-ray spectrum is dominated by a broad Fe $\mathrm{K}$ emission line at $6.7 \mathrm{keV}$ from the inner disk $\left(<255 r_{G}\right)$ during the low-hard state, and by a highly ionized absorption feature with a P Cygni profile, indicating the presence of a disk wind, during the high-soft state. Intriguingly, as the fraction of hard X-ray flux in the jet decreases the strength of the wind increases, as measured by the EW of the absorption feature (Neilsen \& Lee 2009). The latter authors conclude that "it appears that carrying a significant amount of matter away from the accretion disk, strong winds can suppress jet production." The physical process underlying this complex interaction is unknown.

Assuming that the same fundamental processes are at work in stellar-mass and supermassive black holes (e.g., Merloni et al. 2003, and references therein), it is tempting to extend this scenario to AGN. We already know that there is a strong link between the disk and the jet in AGN, in terms of the jet carrying a power similar to that released through the accretion process (Marscher et al. 2002; Maraschi \& Tavecchio 2003; Sambruna et al. 2006). We thus speculate that, similarly to the states of GRS $1915+105$, the formation of powerful, collimated jets in BLRGs subtracts mass and energy that in Seyferts is deposited in the black holes surroundings by the disk wind and manifests itself as the warm absorber in X-ray observations of type- 1 sources. If this scenario is correct, observationally one may naively expect a continuum of X-ray properties across BLRGs and Seyferts as a function of radio power, as hinted at by the limited amount of Suzaku data available so far. This will be easy to verify in the near future using sizable samples of both BLRGs and Seyferts observed with Suzaku.

\subsection{The Jet Contribution in 3 C 390.3}

To investigate in more detail the contribution of the nonthermal jet emission to the SED of 3C 390.3, we present in Figure 7 a set of models which represent the expected jet emission for typical parameters, as observed in blazars. Because of the small beaming factor $(\delta=0.6-1.7$, Section 2$)$, only the external jet regions on pc scales are assumed to be contributing. At this distance from the core, the dominant energy production mechanisms are synchrotron (from radio to optical-UV) and self-Compton scattering (at higher energies), as 3C 390.3 is too nearby for inverse Compton scattering on the cosmic microwave background (CMB) to be energetically important. We used the code of Tavecchio et al. (1998) to compute the models in Figure 7. Briefly, we assumed a spherical emission region with radius $R=10^{17} \mathrm{~cm}$, magnetic field $B=0.3$ $\mathrm{G}$, and a power-law energy distribution of the relativistic electrons with normalization $K=2.3 \times 10^{6} \mathrm{~cm}^{-3}, \gamma_{\min }=50$, 
and slope $n=2.7$ as observed in the extended Chandra jets of FRIIs (e.g., Sambruna et al. 2004). The beaming factor, for $\Gamma_{L}=2$, is $\delta=1.75$, and represents the maximum Doppler factor for a jet inclination angle of $33^{\circ}$. Thus, the models shown in Figure 7 represent the case of amplification of the jet emission in 3 C 390.3 .

The different curves in Figure 7 correspond to different values of the electron energy $\gamma_{\max }$ (see caption). Comparing the theoretical curves to the SED, it can be seen that during averageto-high flux states the predicted jet emission is negligible and the SED is dominated by the accretion-related radiation from IR to hard X-ray wavelengths. However, during faint intensity states of accretion the jet may become a substantial contributor in the optical to medium-soft X-ray band.

Provided that the accretion disk emission rolls off before reaching the gamma-ray band, the jet will be the sole emitter at $\mathrm{GeV}$ energies, where the source can be easily detected with the Fermi Gamma-Ray Space Telescope (blue curve in Figure 7), confirming the earlier prediction of Grandi \& Palumbo (2007). A detection of 3C 390.3 at $\mathrm{GeV}$ energies was not possible with EGRET, whose sensitivity was insufficient as shown in Figure 7. Signatures of a possible jet emission at $\mathrm{GeV}$ will be a soft $\left(\Gamma_{\text {LAT }} \sim 2\right)$ continuum, perhaps with detectable variability on longer timescales ( $\gtrsim$ weeks-months).

\section{CONCLUSIONS}

XMM-Newton and Suzaku observations of the BLRG 3C 390.3 revealed a broadband X-ray spectrum dominated by emission from an accretion flow, with thermal Comptonization mainly responsible for the production of the $2-10 \mathrm{keV}$ continuum. Both cold and ionized reflections appear in the X-ray spectrum; for an assumed $r^{-3}$ emissivity profile and $26^{\circ}$ inclination angle, the ionized emission is consistent with originating from a region that is no less than $\sim 20 r_{G}$ from the central black hole. If true, this suggests that the innermost regions of the disk are obscured, perhaps by the base of the jet, or/and suffer poor illumination. The $\mathrm{Fe} \mathrm{K} \alpha$ line at $6.4 \mathrm{keV}$ is narrow with FWHM $\sim 8800 \mathrm{~km} \mathrm{~s}^{-1}$ and it probably originates from the optical region of the outer disk that emits the optical Balmer lines.

There is no evidence for absorption features in the RGS spectrum of 3C 390.3, consistent with the suggestion that BLRGs lack warm absorbers that are instead common in Seyferts. If confirmed by analysis of larger samples, this would be one of the main observational differences between the two AGN classes, perhaps related to mechanisms of jet-disk wind formation.

Analysis of the multiwavelength SED reveals large-amplitude variability at optical-to-X-rays. We show that the SED is generally dominated by emission from the accretion flow, except during low accretion states, where synchrotron and self-Compton emission from the nonthermal jet can contribute significantly to the optical-UV and X-ray flux, respectively. If the accretion-powered continuum declines around hundreds of $\mathrm{keV}$, as measured with Suzaku and BAT, the jet emission will be the only contributor at $\mathrm{GeV}$ energies, where it can be easily detected with Fermi within a few years of operations.

This research has made use of data obtained from the High Energy Astrophysics Science Archive Research Center (HEASARC), provided by NASA's Goddard Space Flight Center, and of the NASA/IPAC Extragalactic Database (NED) which is operated by the Jet Propulsion Laboratory, California Institute of Technology, under contract with the
National Aeronautics and Space Administration. R.M.S. acknowledges support from NASA through the Suzaku and XMMNewton programs. We thank Ski Antonucci and G. Ghisellini for interesting e-discussions.

Research by A.J.B. is supported by NSF grant AST-0548198. We thank Jenny Greene for assistance with the Keck observations.

Some of the data presented herein were obtained at the W.M. Keck Observatory, which is operated as a scientific partnership among the California Institute of Technology, the University of California and the National Aeronautics and Space Administration. The Observatory was made possible by the generous financial support of the W.M. Keck Foundation. The authors recognize and acknowledge the very significant cultural role and reverence that the summit of Mauna Kea has always had within the indigenous Hawaiian community. We are most fortunate to have the opportunity to conduct observations from this mountain.

\section{APPENDIX}

\section{THE XIS SERENDIPITOUS SOURCE}

A serendipitous source was detected in the XIS field of view at 8.5 from 3C 390.3 with R.A.(2000) = 1844 58, decl.(2000) $=794833$, and a combined XIS $0+3$ count rate of 0.215 counts $\mathrm{s}^{-1}$. This source was not detected in previous X-ray or optical images of the field, suggesting it may be a very fast transient. The XIS spectrum of the source can be characterized by a broken power law with $\Gamma_{1}=2.98 \pm 0.13$ below $2 \mathrm{keV}$ and $\Gamma_{2}=2.12 \pm 0.06$ above $2 \mathrm{keV}$, absorbed by a column density of $N_{\mathrm{H}}=(1.4 \pm 0.2) \times 10^{21} \mathrm{~cm}^{-2}$. The XIS fluxes in the 2-10 and $0.5-10 \mathrm{keV}$ are $3.5 \times 10^{-12} \mathrm{erg} \mathrm{cm}^{-2} \mathrm{~s}^{-1}$ and $2.8 \times 10^{-12} \mathrm{erg} \mathrm{cm}^{-2} \mathrm{~s}^{-1}$. If we extrapolate this model into the HXD/PIN bandpass, then the expected flux in the 15$50 \mathrm{keV}$ band is $1.7 \times 10^{-12} \mathrm{erg} \mathrm{cm}^{-2} \mathrm{~s}^{-1}$. This is $3 \%$ of the $15-50 \mathrm{keV}$ flux of $3 \mathrm{C} 390.3\left(5.4 \times 10^{-11} \mathrm{erg} \mathrm{cm}^{-2} \mathrm{~s}^{-1}\right)$. Thus, the XIS serendipitous source, being quite soft, is not expected to contaminate the HXD spectrum of 3C 390.3.

\section{REFERENCES}

Alef, W., Preuss, E., \& Kellerman, K. I. 1988, A\&A, 192, 53

Alef, W., Wu, S. Y., Preuss, E., Kellermann, K. I., \& Qiu, Y. H. 1996, A\&A, 308, 376

Ballantyne, D. R. 2005, MNRAS, 362, 1183

Ballantyne, D. R. 2007, Mod. Phys. Lett. A, 22, 2397

Ballantyne, D. R., Fabian, A. C., \& Iwasawa, K. 2004, MNRAS, 354, 839

Ballantyne, D. R., Ross, R. R., \& Fabian, A. C. 2002, MNRAS, 332, L45

Balzano, V. A., \& Weedman, D. W. 1981, ApJ, 243, 756

Bentz, M. C., Peterson, B. M., Pogge, R. W., Vestergaard, M., \& Onkon, C. A. 2006, ApJ, 644, 133

Bird, A. J., et al. 2007, ApJS, 170, 175

Blandford, R. D. 1985, in Active Galactic Nuclei, ed. J. E. Dyson (Manchester: Manchester Univ. Press), 281

Boldt, E. 1987, Phys. Rep., 146, 215

Dietrich, M., et al. 1998, ApJS, 115, 185

Eracleous, M., \& Halpern, J. P. 2003, ApJ, 599, 886

Eracleous, M., \& Halpern, J. P. 1994, ApJS, 90, 1

Eracleous, M., Halpern, J. P., \& Livio, M. 1996, ApJ, 459, 89

Eracleous, M., Sambruna, R. M., \& Mushotzky, R. F. 2000, ApJ, 537, 654

Evans, D. A., Worrall, D. M., Hardcastle, M. J., Kraft, R. P., \& Birkinshaw, M. 2006, ApJ, 642, 96

Fabian, A. C., et al. 2002, MNRAS, 335, L1

George, I. M., \& Fabian, A. C. 1991, MNRAS, 249, 352

George, I. M., et al. 1998, ApJS, 114, 73

Gezari, S., Halpern, J. P., \& Eracleous, M. 2007, ApJS, 169, 167

Ghisellini, G., Haardt, F., \& Matt, G. 2004, A\&A, 413, 535

Ghisellini, G., \& Madau, P. 1996, MNRAS, 280, 67 
Giovannini, G., Cotton, W. D., Feretti, L., Lara, L., \& Venturi, T. 2001, ApJ, 552,508

Gliozzi, M., Sambruna, R. M., \& Eracleous, M. 2003, ApJ, 584, 176

Gliozzi, M., et al. 2009, ApJ, submitted

Grandi, P., Guainazzi, M., Cappi, M., \& Ponti, G. 2007, MNRAS, 381, L21

Grandi, P., Guainazzi, M., Haardt, F., Maraschi, L., Massaro, E., Matt, G., Piro, L., \& Urry, C. M. 1999, A\&A, 343, 33

Grandi, P., Malaguti, G., \& Fiocchi, M. 2006, ApJ, 642, 113

Grandi, P., \& Palumbo, G. G. C. 2007, ApJ, 659, 235

Grandi, P., Urry, C. M., \& Maraschi, L. 2002, New Astron. Rev., 46, 221

Guainazzi, M., Fabian, A. C., Iwasawa, K., Matt, G., \& Fiore, F. 2005, MNRAS, 356,295

Heckman, T. M., Lebofsky, M. J., Rieke, G. H., \& van Breugel, W. 1983, ApJ, 272,400

Ho, L. C. 1999, ApJ, 516, 672

Junor, W., Biretta, J. A., \& Livio, M. 1999, Nature, 401, 891

Kalberla, P. M. W., et al. 2005, A\&A, 440, 775

Kataoka, J., et al. 2007, PASJ, 59, 279

Kokubun, M., et al. 2007, PASJ, 59, 53

Larsson, J., Fabian, A. C., Ballantyne, D. R., \& Miniutti, G. 2008, MNRAS, 388, 1037

Leighly, K. M., \& O’Brien, P. T. 1997, ApJ, 481, 15

Leighly, K. M., et al. 1997, ApJ, 483, 767

Lewis, K. T., \& Eracleous, M. 2006, ApJ, 642, 711

Madrid, J. P., et al. 2006, ApJS, 164, 307

Malaguti, G., Bassani, L., \& Caroli, E. 1994, ApJS, 94, 517

Maraschi, L., \& Tavecchio, F. 2003, ApJ, 593, 667

Martin, D. C., et al. 2005, ApJ, 619, L1

Marscher, A. P., Jorstad, S. G., Gomez, J.-L., Aller, M., Terasranta, H., Lister, M., \& Stirling, A. 2002, Nature, 417, 625

Merloni, A., Heinz, S., \& Di Matteo, T. 2003, MNRAS, 345, 1057

Miley, G., Neugebauer, G., Soifer, B. T., Clegg, P. E., Harris, S., RowanRobinson, M., \& Young, E. 1984, ApJ, 278, L79

Nandra, K., O’Neill, P. M., George, I. M., \& Reeves, J. N. 2007, MNRAS, 382, 194

Narayan, R. 2005, Ap\&SS, 300, 177

Narayan, R., Mahadevan, R., \& Quataert, E. 1998, in Theory of Black Hole Accretion Disks, ed. Marek A. Abramowicz, G. Bjornsson, \& James E. Pringle (Cambridge: Cambridge Univ. Press), 148

Neilsen, J., \& Lee, J. C. 2009, Nature, 458, 481

Nelson, C. H., Green, R. F., Bower, G., Gebhardt, K., \& Weistrop, D. 2004, ApJ, 615,654
O’Brien, P., et al. 1998, ApJ, 509, 163

Ogle, P., Wysong, D., \& Antonucci, R. R. J. 2006, ApJ, 647, 161

Ogle, P., et al. 2000, ApJ, 618, 139

Pearson, T. J., \& Readhead, A. C. S. 1988, ApJ, 328, 114

Pollock, A. M. T. 2008, Status of the RGS Calibration (Noordwijk: ESA), http://xmm2.esac.esa.int/docs/documents/CAL-TN-0030-5-1.pdf

Ponti, G., et al. 2009, MNRAS, 394, 1487

Rees, M. J., Begelman, M. C., Blandford, R. D., \& Phinney, E. S. 1982, Nature, 295, 17

Reeves, J. N., \& Turner, M. J. L. 2000, MNRAS, 316, 234

Reeves, J. N., et al. 2007, PASJ, 59, 301

Reynolds, C. S., Nowak, M. A., Markoff, S., Tueller, J., Wilms, J., \& Young, A. J. 2009, ApJ, 691, 1159

Ross, R. R., \& Fabian, A. C. 2005, MNRAS, 358, 211

Sambruna, R. M., Gliozzi, M., Tavecchio, F., Maraschi, L., \& Foschini, L. 2006, ApJ, 652, 456

Sambruna, R. M., Eracleous, M., \& Mushotzky, R. 1999, ApJ, 526, 60

Sambruna, R. M., Eracleous, M., \& Mushotzky, R. F. 2002, New Astron. Rev., 46, 215

Sambruna, R. M., Reeves, J. N., \& Braito, V. 2007, ApJ, 665, 1030

Sambruna, R. M., et al. 2004, ApJ, 608, 698

Schlegel, D. J., Finkbeiner, D. P., \& Davis, M. 1998, ApJ, 500, 525

Seaton, M. J. 1979, MNRAS, 187, 83P

Sheinis, A. I., Bolte, M., Epps, H. W., Kibrick, R. I., Miller, J. S., Radovan, M. V., Bigelow, B. C., \& Sutin, B. M. 2002, PASP, 114, 851

Siebenmorgen, R., Freudling, W., Krugel, E., \& Haas, M. 2004, A\&A, 421, 129 Spergel, D. N., et al. 2003, ApJS, 148, 175

Steffen, A. T., Strateva, I., Brandt, W. N., Alexander, D. M., Koekemoer, A. M., Lehmer, B. D., Schneider, D. P., \& Vignali, C. 2006, AJ, 131, 2826

Steppe, H., Salter, C. J., Chini, R., Kreysa, E., Brunswig, W., \& Lobato Perez, J. 1998, A\&AS, 75, 317

Strüder, L., et al. 2001, A\&A, 365, L18

Tavecchio, F., Maraschi, L., \& Ghisellini, G. 1998, ApJ, 509, 608

Tavecchio, F., et al. 2000, ApJ, 543, 535

Tueller, J., et al. 2008, ApJ, 681, 113

Turner, T. J., Miller, L., Kraemer, S. B., Reeves, J. N., \& Pounds, K. A. 2009, ApJ, 698, 99

Turner, M. J. L., et al. 2001, A\&A, 365, L27

Veilleux, S., \& Zheng, W. 1991, ApJ, 377, 89

Zdziarski, A. A., \& Grandi, P. 2001, ApJ, 551, 186

Zheng, W. 1996, AJ, 111, 1498

Zirbel, E., \& Baum, S. 1998, ApJS, 114, 177 